1 Glial immune-related pathways as mediators of closed head TBI effects on behavior in

\title{
2 Drosophila
}

4 Bart van Alphen ${ }^{1 *}$, Samuel Stewart ${ }^{1,2 *}$, Marta Iwanaszko ${ }^{1,3}$, Fangke $\mathrm{X}^{1}{ }^{1}$, Eugenie Bang ${ }^{1}$, Sydney

5 Rozenfeld ${ }^{1}$, Anujaianthi Ramakrishnan ${ }^{1}$, Taichi Q. Itoh ${ }^{1,4}$, Rosemary, I. Braun ${ }^{3,5}$, Ravi Allada ${ }^{1}$

$7 \quad{ }^{1}$ Department of Neurobiology, Northwestern University, Evanston, IL 60208, USA

$8{ }^{2}$ Current affiliation: Department of Neuroscience, Baylor College of Medicine, Houston, TX,

977030

$10{ }^{3}$ Department of Preventive Medicine - Biostatistics, Feinberg School of Medicine, Northwestern

11 University, Chicago, $60611 \mathrm{IL}, \mathrm{USA}$

$12{ }^{4}$ Current affiliation: Faculty of Arts and Science, Kyushu University, 744 Motooka, Nishi-ku,

13 Fukuoka, 819-0395, Japan

$14{ }^{5}$ Department of Engineering Sciences and Applied Mathematics, Northwestern University,

15 Chicago, 60208 IL, USA

16

17 *Authors contributed equally 


\section{Abstract}

21 In traumatic brain injury (TBI) the initial injury phase is followed by a secondary phase that

22 contributes to neurodegeneration. Yet the mechanisms leading to neuropathology in vivo remain

23 to be elucidated. To address this question, we developed a Drosophila head-specific model for

$24 \mathrm{TBI}$, which we term Drosophila Closed Head Injury $(\mathrm{dCHI})$, where well-controlled, non-

25 penetrating strikes are directly delivered to the head of unanesthetized flies. This assay

26 recapitulates many TBI phenotypes, including increased mortality, impaired motor control,

27 fragmented sleep, and increased neuronal cell death. To discover novel mediators of TBI, we

28 used glial targeted translating ribosome affinity purification in combination with RNA

29 sequencing. We detected significant changes in the transcriptome at various times after TBI

30 including in genes involved in innate immunity within 24 hours after TBI. To test the in vivo

31 functional role of these changes, we examined TBI-dependent behavior and lethality in mutants

32 of the master immune regulator NF-KB and found that while lethality effects were still evident,

33 changes in sleep and motor function were substantially reduced. These studies validate a new

34 head-specific model for TBI in Drosophila and identify glial immune pathways as candidate in

35 vivo mediators of TBI effects. 
38 Traumatic brain injury ( $\mathrm{TBI}$ ) is one of the leading causes of death and disability in the developed 39 world [1-3]. Yet the underlying mechanisms that lead to long term physical, emotional, and

40 cognitive impairment remain unclear.

42 Unlike in most forms of trauma, a large percentage of people killed by traumatic brain injuries do 43 not die immediately but rather days or weeks after the insult [4]. TBI consists of a primary and a

44 secondary phase. The primary brain injury is the result of an external mechanical force,

45 resulting in damaged blood vessels, axonal shearing [5], cell death, disruption of the blood-

46 brain barrier, edema, and the release of damage associated molecular patterns (DAMPs) and

47 excitotoxic agents [6]. In response, local glia and infiltrating immune cells upregulate cytokines

48 (tumor necrosis factor $\alpha$ ) and interleukins (IL-6 and IL-1 $\beta$ ) that drive post-traumatic

49 neuroinflammation [7-10]. This secondary injury develops over a much longer time course,

50 ranging from hours to months after the initial injury and is the result of a complex cascade of

51 metabolic, cellular and molecular processes [11-13]. Neuroinflammation is beneficial when it is

52 promoting clearance of debris and regeneration [14] but can become harmful, mediating

53 neuronal death, progressive neurodegeneration, and neurodegenerative disorders [15-18]. The

54 mechanisms underlying these opposing outcomes are largely unknown, but are thought to

55 depend of the location and timing of the neuroinflammatory response $[19,20]$. It remains to be

56 determined what the relative roles of TBI-induced neuroinflammation and other TBI-induced

57 changes are in mediating short and long-term impairments in brain function in vivo.

59 To study the mechanisms that mediate TBI pathology in vivo over time, we employ the fruit fly

60 Drosophila melanogaster, a model organism well suited to understanding the in vivo genetics of

61 brain injury. Despite considerable morphological differences between flies and mammals, the

62 fly brain operates on similar principles through a highly conserved repertoire of neuronal

63 signaling proteins, including a large number of neuronal cell adhesion receptors, synapse- 
64 organizing proteins, ion channels and neurotransmitter receptors, and synaptic vesicle-

65 trafficking proteins [21].This homology makes Drosophila a fruitful model to study

66 neurodegenerative disorders [22], including ALS [23], Alzheimer's disease [24], Huntington's

67 disease [25] and Parkinson's disease [26].

69 Trauma-induced changes in glial gene expression are a highly conserved feature of both

70 mammalian [27, 28] and Drosophila glia [29-32] (reviewed in [33]). In Drosophila, glia are able to

71 perform immune-related functions [32, 34]. Ensheathing glia can act as phagocytes and

72 contribute to the clearance of degenerating axons from the fly brain [29, 31, 35]. The Drosophila

73 innate immune system is highly conserved with that of mammals and consists primarily of the

74 Toll, Immunodeficiency (Imd) and Janus Kinase protein and the Signal Transducer and Activator

75 of Transcription (JAK-STAT) pathways, which together combat fungal and bacterial infections

$76[36,37]$. Dysregulation of cerebral innate immune signaling in Drosophila glial cells can lead to

77 neuronal dysfunction and degeneration [38, 39], suggesting that changes in glia cells could

78 underlie secondary injury mechanisms in our Drosophila model of TBI.

80 Existing Drosophila TBI models [40, 41] deliver impacts to the entire body, not just the head,

81 and thus, one cannot definitively attribute ensuing phenotypes to TBI. To remove the confound

82 of bodily injury, we have developed a novel, head-specific Drosophila model for TBI, Drosophila

83 Closed Head Injury (dCHI). Here we show that by delivering precisely controlled, non-

84 penetrating strikes to an unanesthetized fly's head, we can induce cell death and increased

85 mortality in a dose-dependent manner. In addition, TBI results in impaired motor control and

86 decreased, fragmented sleep. Impaired motor control persists for many days after TBI while the

87 sleep phenotype disappears after three days. These TBI-induced behavioral phenotypes do not

88 occur in mutants lacking the master immune regulator NF-kB Relish (ReI), even though TBI-

89 induced mortality is greatly induced in these mutants. In wild type flies, TBI results in changes in 
90 glial gene expression, where many immune related genes are upregulated 24 hours after injury.

91 Together, these results establish a platform where powerful Drosophila genetics can be utilized

92 to study the complex cascade of secondary injury mechanisms that occur after TBI in order to

93 genetically disentangle its beneficial and detrimental effects.

94

95 Methods

96 Flies

97 Fly stocks were raised on standard cornmeal food under a $12 \mathrm{~h} \mathrm{light} / 12 \mathrm{~h}$ dark cycle at $25^{\circ} \mathrm{C}$ and

$98 \sim 65 \%$ relative humidity. TBI inductions and climbing assays were carried out in the lab at room

99 temperatures $\left(\sim 21-23^{\circ} \mathrm{C}\right)$. For sleep and lifespan experiments, flies were kept on standard

100 cornmeal food under a $12 \mathrm{~h}$ light $/ 12 \mathrm{~h}$ dark cycle at $25^{\circ} \mathrm{C}$ and $\sim 65 \%$ relative humidity. All

101 experiments were carried out in young adult $w^{1118}$ males that are 3-7 days old. NF-KB Relish

102 null mutants (Relish[E20]) were obtained from Bloomington (w ${ }^{1118} ;$ Rel $^{[\mathrm{E} 20]} \mathrm{e}^{[\mathrm{s}]}$; \#9457). Repo-

103 Gal4 was obtained from Bloomington (w[1118]; $\left.P\left\{w\left[+m^{*}\right]=G A L 4\right\} r e p o / T M 3, S b[1] \# 7415\right)$. UAS-

104 GFP::RpL 10A was obtained from the Jackson lab [42]. All flies were collected under $\mathrm{CO}_{2}$

105 anesthesia at least 24 hours before TBI induction and placed on regular food.

107 Aspirator and fly restraint assembly

108 Aspirators were constructed by wrapping a small square of cheesecloth around one end of

109 aquarium tubing. A P1000 pipette tip was securely attached to covered end of the tubing, and

110 the tip of the pipette tip was cut off to leave an aperture large enough for an individual fly to pass

111 through without difficulty. The aspirator is used to transport individual flies via mouth pipetting.

112 This allows flies to be transferred from their home vials to the experimental setup without using

113 anesthesia. Fly restraints were created by cutting off the last 3-4 millimeters of P200 pipette tips

114 to create an aperture large enough to let an individual fly's head through without letting the 
115 entire body through. Multiple sizes of fly restraints were produced, to accommodate small

116 variations in size among flies.

Drosophila closed head TBI assay

119 Flies were removed from their home vials without the use of anesthetic, using an aspirator and

120 gently transferred to a prepared P200 pipette (see above). By applying some air pressure on the

121 aspirator, the fly is pushed into the P200 pipette in such a way that the fly gets stuck at the end,

122 with only its head sticking out. The restrained fly is then placed in a micromanipulator allowing

123 for movement in three dimensions, which was subsequently used to move the fly into the

124 appropriate position, with the back of the fly's head making contact with the pin of a pull-type

125 solenoid (uxcell DC 12V) which delivers 8.34 Newtons of force. Flies were observed using a

126 high-powered camera lens (Navitar Zoom 6000, Rochester, NY) to ensure that they were in the

127 proper position. A variable-voltage power supply (Tenma Corporation, Tokyo, Japan) was set to

$12812 \mathrm{~V}$ and used to power the solenoid, which then delivered a blow to the fly's head (Fig. 1). Flies

129 were hit 1 time, 5 times, and 10 times when observing effect of number of blows on response to

130 TBI. Flies were hit 5 times for all other experiments. To minimize confounding effects of

131 anesthesia, flies were collected under $\mathrm{CO}_{2}$ anesthesia at least 24 hours before each

132 experiment. All experiments are carried out in awake, unanesthesized flies.

134 Negative geotaxis response

135 A climbing assay is used to measure locomotor deficits after TBI in a manner similar to the

136 RING assay [43]. Flies were individually stored in food vials and kept under the conditions

137 discussed above. Vials were vertically divided into six 1-cm tall segments, labeled in order of

138 ascending height $(0 \mathrm{~cm}, 1 \mathrm{~cm}$, etc.). Vials were tapped on a lab bench as a startle stimulus.

139 Flies were then allowed to climb freely for 4 seconds, after which the highest point reached by

140 the flies was observed and recorded. Three trials were observed for each individual fly; flies 
141 were allowed a period of at least 1 minute of recovery in between trials. Measurements from

142 individual flies' trials were then averaged to calculate a fly's mean performance.

\section{Mortality assay}

145 After TBI induction, flies are housed in plastic vials with standard corn meal medium and housed

146 in a $12 \mathrm{~h}$ light $/ 12 \mathrm{~h}$ dark cycle at $25^{\circ} \mathrm{C}$ and $\sim 65 \%$ relative humidity. Flies are gently transferred to

147 fresh vials every three days. Deceased flies remaining in the old vial are counted.

149 Sleep assay

$150 \quad 3-7$ days old flies were placed into individual 65-mm glass tubes in the Drosophila activity

151 monitoring (DAM) system (Trikinetics, Waltham, MA), which were placed in incubators running a

$15212 \mathrm{~h}$ light $/ 12 \mathrm{~h}$ dark cycle. All experiments were carried out at $25^{\circ} \mathrm{C}$. Sleep data was collected by

153 the DAM system in 1-minute bins and analyzed offline using custom made Matlab scripts

154 (Matlab 2011a, Mathworks, Natick, MA). Briefly, sleep was defined as any period of inactivity of

155 five minutes or more [44, 45]. For each fly, total amount of sleep per day, average bout length,

156 number of sleep bouts, number of brief awakenings and average daily activity were derived from

157 its activity trace (number of infrared beam crossings per minute).

Statistics

160 All statistical analysis for behavioral experiments was performed using Matlab 2011a for PC. For

161 TRAP-seq analysis, see below. Mortality assay: Survival curves were plotted using the Kaplan-

162 Meier estimator as described [46]. The statistical significance was calculated using the log rank

163 test. Plots and log rank tests were performed in Matlab, using scripts developed by [47]. 
168 A TUNEL assay was performed in whole brain as per manufacturer's protocol (In situ cell death

169 detection kit, Fluorescein, Sigma Aldrich). The brains were carefully dissected out at different

170 time points and fixed in 4\% paraformaldehyde for 20 min followed by $3 \times 15$ min wash in PBST

171 (PBS with 0.5\% Triton-X 100). The brains were incubated in TUNEL mixture (prepared as per

172 manufacturer's instruction) for $60 \mathrm{~min}$ at $37^{\circ} \mathrm{C}$ followed by $3 \times 15 \mathrm{~min}$ wash in PBST. The brains

173 were then mounted in Vectashield mounting medium.

175 TRAP-seq

176 After receiving TBI, flies were collected at one of three time points, namely, 1 day post-injury, 3

177 days post-injury, and 7 days post-injury at ZTO (lights-on in $12 \mathrm{~h}$ light: $12 \mathrm{~h}$ dark). Flies were

178 collected in $15 \mathrm{ml}$ conical tubes, and flash frozen in liquid nitrogen. Their heads were collected

179 by vigorously shaking frozen flies and passing them through geological sieves. Approximately

180100 heads were used for each experiment. Translating Ribosome Affinity Purification and

181 Sequencing (TRAP-Seq) was performed as described [42, 48]. Sepharose beads were

182 prepared by rinsing $25 \mu \mathrm{L}$ of resin per reaction with $1 \mathrm{~mL}$ of extraction buffer. Protein A Plus

183 UltraLink (PAS) resin was incubated with $1 \mathrm{~mL}$ of extraction buffer and $2.5 \mathrm{uG}$ of HTZ 19C8

184 antibody and rotated for 2-3 hours at room temperature. Beads were then spun at $2500 \mathrm{~g}$ for 30

185 seconds at room temperature and rinsed another 3 times with extraction buffer. The conjugated

186 beads were then incubated with $1 \mathrm{~mL}$ of blocking buffer for 15 minutes at $4^{\circ} \mathrm{C}$. The beads were

187 then spun again at $2500 \mathrm{~g}$ for 30 seconds, and the supernatant was discarded. The beads were

188 washed with $1 \mathrm{ml}$ cold extraction buffer. This process was repeated another two times. Beads

189 were incubated with $260 \mu \mathrm{L}$ of head extract for 1 hour at $4^{\circ} \mathrm{C}$, and then spun at $2500 \mathrm{~g}$ for 30

$190 \mathrm{sec}$ at $4^{\circ} \mathrm{C}$. The beads were rinsed with $1 \mathrm{~mL}$ of cold wash buffer at $4^{\circ} \mathrm{C}$. This process was

191 repeated 3 times. After the final wash, $1 \mathrm{~mL}$ of Trizol was added. The beads were rotated at

192 room temperature for 15 minutes. Chloroform was added, and the beads were subsequently 
shaken by hand for 30 seconds and incubated for 3 minutes at room temperature. The beads were then centrifuged at $15,000 \mathrm{rpm}$ for 15 minutes at $4^{\circ} \mathrm{C}$. The resulting upper aqueous phase was extracted and transferred to a new tube with $70 \%$ ethanol. RNA was extracted following the RNeasy Micro Kit protocol (Qiagen, Venlo, Netherlands). RNA purified from the GFP tagged RpL10 was then reverse transcribed to cDNA. The cDNA was used as template for T7 transcriptase to amplify the original RNA. Second round cDNA was synthesized based on amplified RNA. Detailed procedure for amplification can be in found in [49]. cDNA was sent to High Throughput Genome Analysis Core (HGAC) at the University of Chicago for library preparation and sequencing. Control and TBI samples were provided in three replicates.

\section{Quantification of data, differential expression and functional annotation analyses}

204 Sequencing was done with Illumina HiSeq 2000. All samples are done with single end reads of 50 base pairs in length. At least 5,000,000 mappable reads were obtained and used for quantification for each sample. RNA-seq data were quantified at transcript level using Kallisto [50], with FlyBase_r6.14 as a reference transcriptome [51]. Quantified transcripts were summed up to the gene level using tximport library [52]. A minimal pre-filtering, keeping only rows with more than two reads, was applied to gene level data before differential expression (DE) analysis. Differential gene expression analysis was performed on TBI vs. control data with

211 DESeq2 [53], using the likelihood ratio test to correct for batch effect among the biological

212 replicates. Genes with the absolute $\log 2$ fold change higher than 0.6 , and False Discovery Rate 213 adjusted $p$-values $\leq 0.1$ were identified as differentially expressed. Day 7 replicates were

214 corrected for sequencing depth and possibly other distributional differences between lanes,

215 using upper-quartile (UQ) normalization, available through RUVSeq library [54], before

216 proceeding to DE analysis. One replicate was removed from further analysis, due to extremely

217 low expression across the sample, which was not comparable to the levels observed in the 218 other Day 7 replicates. Functional annotation of DE genes was performed using the DAVID 
database (release $6.8[55,56])$ with a focus on gene ontology (GO) terms and Reactome pathways.

Supplemental Figures for post-TBI days 1, 3 and 7 (Fig. S2) show sample comparison of relative log expression in untreated and successfully corrected data (panels $A, C, E$ and $B, D, F$ respectively). Small deviations, arising from the technical differences, can be observed in D01 and D03, these were removed with upper-quartile between lane correction [57]. For D07 (Fig. S1E) we have observed that the replicates are lower quality and there is a significant deviation

227 in values between replicates within the TBI group, with replicate R3 assumed to be corrupted 228 (see Supplemental Table 2). For consistency we applied the same correction method to remove technical differences from post-TBI day 7, but as expected, replicate R3 did not improve. Taking this into consideration we decided to remove this replicate from further analysis.

Results

dCHI: A Novel Controlled Head Impact Model for TBI in Drosophila

234 To study TBI in flies, we developed a novel model where brain injury is inflicted in awake,

235 individually restrained flies using a solenoid to deliver well-controlled, non-penetrating strikes to 236 the fly head (Fig. 1). For TBI induction, individual flies are transferred from their home vial to a 237 prepared P200 pipette tip, using an aspirator. Flies are gently blown upward until the head 238 emerges from the tip of the pipette (Fig. 1B). The pipette is then placed in a micromanipulator 239 platform with five degrees of freedom (pitch, roll as well as movement along the XYZ axes). The 240 top of the fly head is pressed against the tip of the solenoid that consists of a metal pin running

241 through a copper coil attached to a spring. By running a current through the coil, it acts as a

242 magnet, drawing the pin back and arming the spring. When the current is halted, the spring 243 causes the pin to shoot out, thus allowing us to deliver one or more blows to the fly's head (Fig. 
244 1C, Sup Movie 1). After TBI induction, flies are aspirated out of the pipette tip and returned to

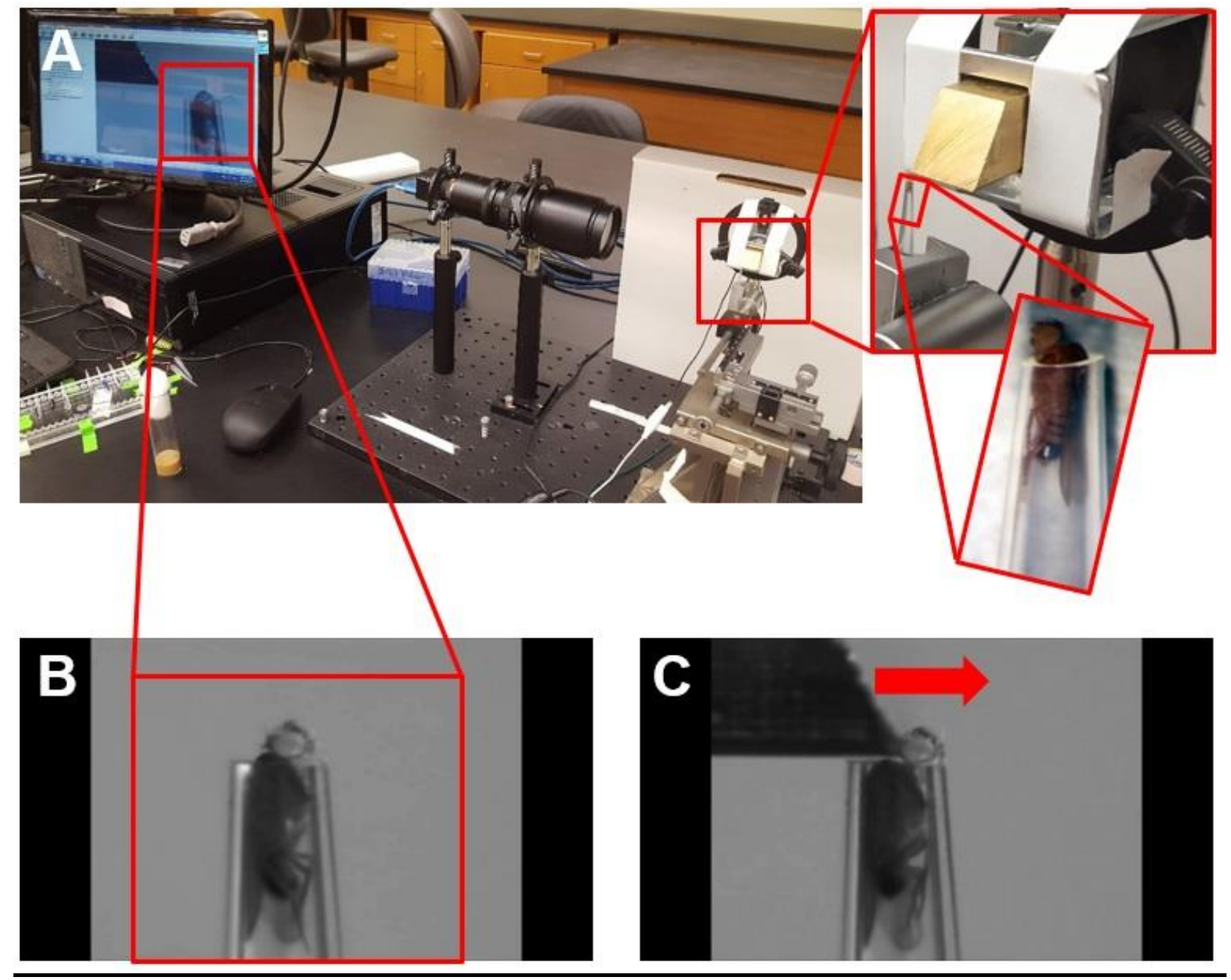

Figure 1: Single fly TBI setup

$247 \boldsymbol{A}, \boldsymbol{B})$ To induce head-specific TBI, individual flies are gently aspirated into a modified 200 $\mu$ l

248 pipette tip that acts as a restraint. Immobilized flies are placed in front of a solenoid, using a set

249 of micromanipulators with 5 degrees of freedom $(x, y, z$, pitch, roll) and a high magnification video

250 system to ensure highly replicable positioning. TBI is induced by running a current through the

251 magnetic coil of the solenoid, which retracts a brass trapezoid-shaped block. C) By releasing

252 current, a spring drives the brass block forward, hitting the fly on the top of the head. 
253 an empty vial containing regular fly food. Immediately after TBI induction, flies often seem

254 dazed, being able to stand but only barely responding to tactile stimuli (Sup. Movie 2). However,

255 mobility returns in a manner of minutes (Sup. Movie 3).

$257 \mathrm{dCHI}$ increases mortality and impairs negative geotaxis in a dose-dependent manner within $24 \mathrm{~h}$

258 To address the impact of $\mathrm{dCHI}$, we first examined the acute pathological and behavioral effects

259 within the first $24 \mathrm{~h}$ post-dCHI. TBI phenotypes become more severe with consecutive strikes in

260 mammals [58] and Drosophila [40, 41]. We subjected male flies to 1, 5 or 10 consecutive

261 solenoid strikes, delivered at 1 strike per second. After TBI induction, treated and sham treated

262 cohorts were individually housed in vials containing standard food. 24 hours after TBI exposure,

263 surviving flies were counted in each of the four groups. We observed a dose dependent

264 increase in 24-hour mortality (Fig. 2A). At 1 strike (TBIx1), there is no effect on 24-hour mortality

$265(p=0.68)$. Mortality is increased in a dose dependent manner (control vs TBIx5, $p=0.03$; control

266 vs TBIx10, $p=0.004$; ANOVA with Dunnett's post hoc test, $F(3,8)=8.41 ; n=$ three replicates of

26710 flies/group).

269 Loss of balance and poor motor coordination are symptoms associated with TBI [59-61].

270 Impairments in motor control, balance and sensorimotor integration are also a well-studied

271 endophenotype in rodent models of TBI (as quantified by beam balance, beam walk and rotarod

272 assays, reviewed in [62]). In Drosophila, impairments in sensorimotor integration are quantified

273 by measuring the negative geotaxis response, a reflexive behavior where a fly moves away

274 from gravity's pull when agitated [63]. Impaired negative geotaxis has been observed in aging

275 and in Drosophila models of neurodegeneration [64-66]. 
A 24 hr survival rate
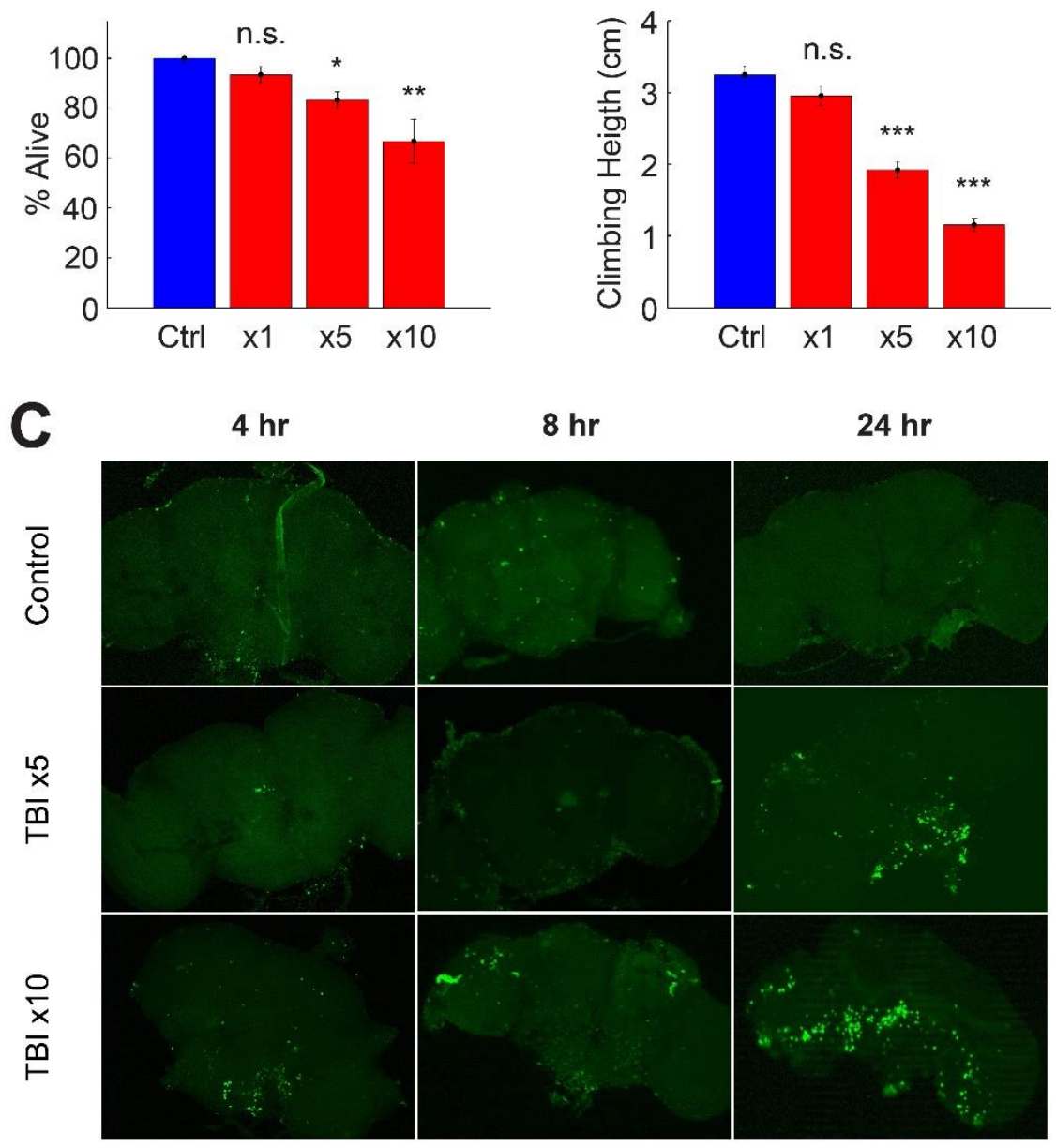

D

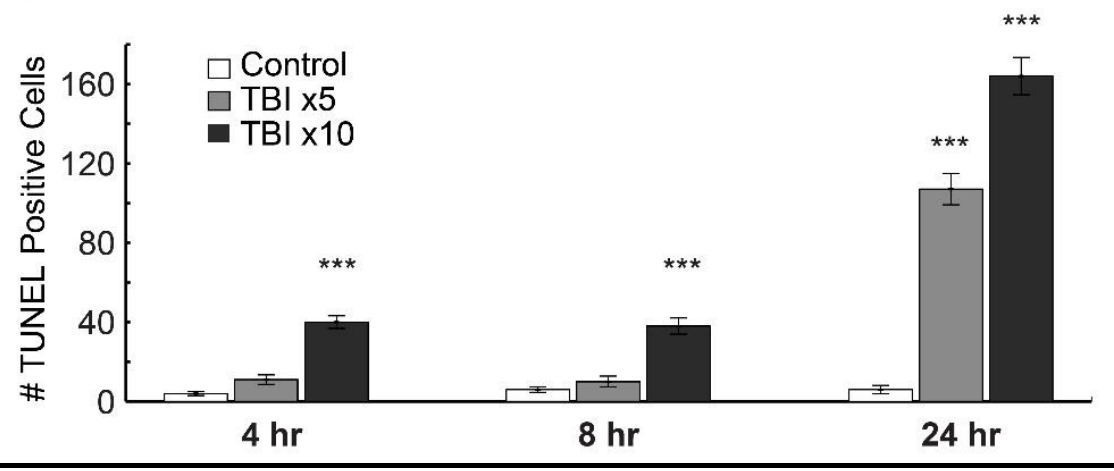

277 Figure 2: TBI causes cell death, mortality and impaired climbing in a dose-dependent

279 Male $w^{1118}$ flies were exposed to either 1,5 or 10 strikes to the head, delivered at 1 strike per

280 second (n=32 per group). A) 24 hour survival rate decreased with increased number of strikes. 
B) in surviving flies, climbing behavior was quantified and compared to sham-treated controls 24 hours after TBI. Climbing behavior became more impaired with increased TBI severity (n.s = not significant, ${ }^{* *} p<0.001$, one-way ANOVA with Dunnett post hoc test, $n=32 /$ group). Cell death following TBI was quantified with a TUNEL assay (C) Representative images of TUNEL staining at different time points in control and post TBI flies (D) Histogram showing significantly increased TUNEL positive cells post TBI in a dose dependent manner ( $n=10,8,8$ for controls, $n=8,8,9$ for TBIx5 and $n=7,7,8$ for TBIx10 at 4hr, 8hr and $24 \mathrm{hr}$ respectively). ${ }^{\star} p<0.05,{ }^{* *} p$ $<0.01,{ }^{* * *} p<0.001$ ANOVA with Dunnett posthoc test. Errorbars indicate SEM.

To assess sensorimotor function after TBI, we used a variation of the negative geotaxis assay

291 [43], where the average height climbed in a defined time period is quantified, rather than a 292 pass/fail number for absolute height as more subtle deficits can be observed using this 293 approach. Typically, young adult wild-type flies reach an average climbing height of $\sim 4-5 \mathrm{~cm}$ in 294 a 3-second time period [43]. In our assay, sham treated $w^{1118}$ flies (3-7 days old males) reached 295 an average height of $\sim 3.4 \mathrm{~cm}$ in four seconds (Fig. 2B, 0 days post-TBI). Climbing behavior, 296 driven by negative geotaxis becomes impaired after TBI. After a single hit, there is no detectable 297 difference in climbing, 24 hours after TBI induction (control vs TBIx1, p=0.1876, Fig. 2B).

298 However, after five or ten consecutive hits, climbing behavior becomes impaired in a dose299 dependent manner (Fig. 2B, control vs TBIx5, $p=2.67 \times 10^{-6}$; control vs TBIx10, $p=2.67 \times 10^{-6}$;

300 ANOVA with Dunnett's post hoc test, $F(3,99)=57.54 ; n=30$ flies/group)

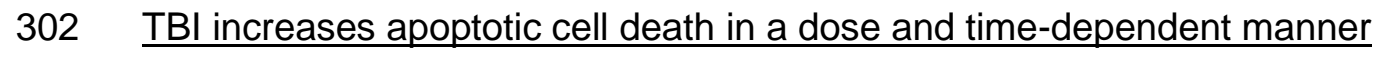

303 To test whether our TBI assay causes neuronal death, apoptosis was quantified using a TUNEL 304 assay [67] after inducing TBI by striking flies either 5 or 10 times and comparing the number of 305 TUNEL positive cells at three different timepoints (4, 8 and 24 hours) between TBI-treated flies 

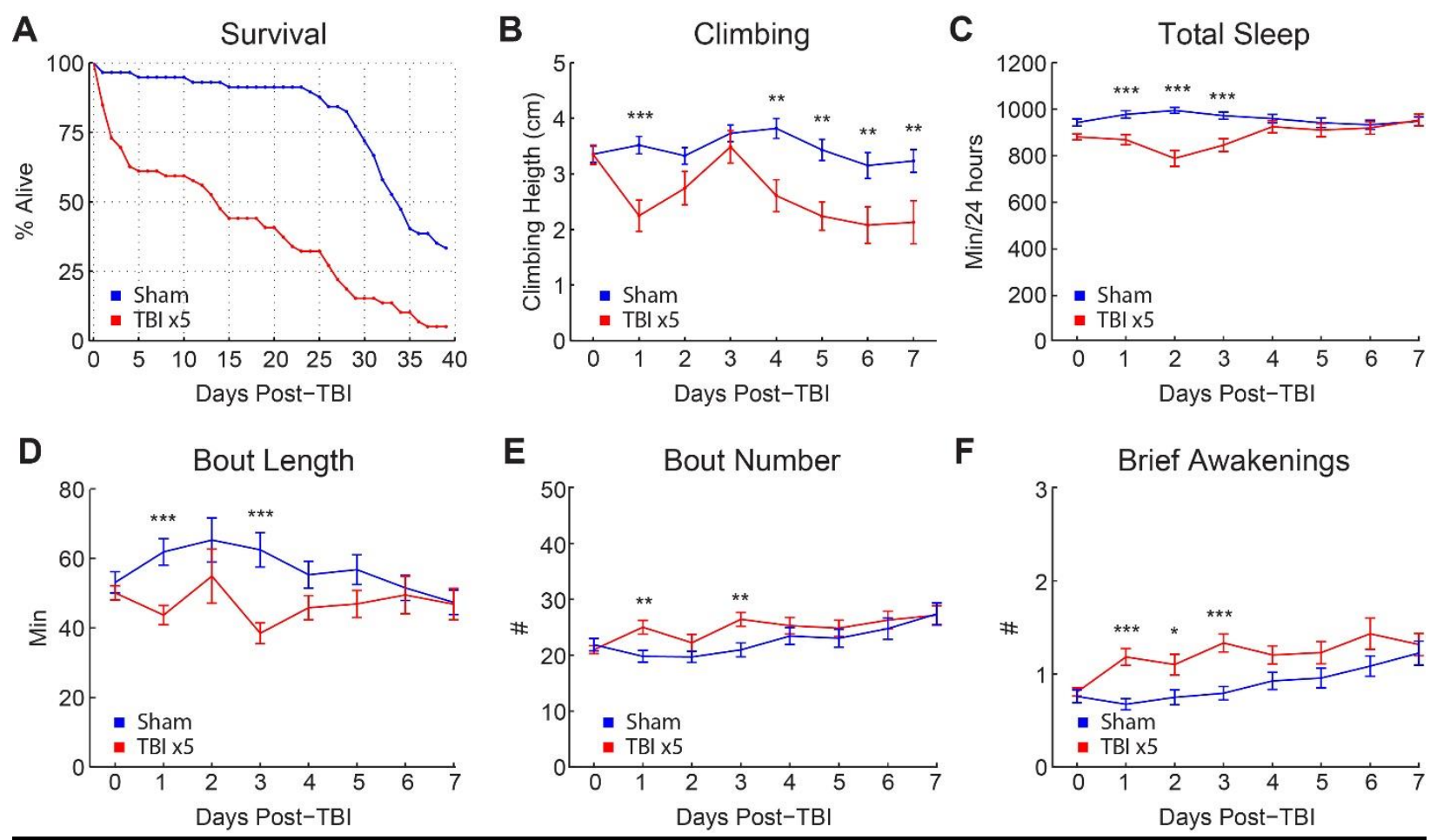

Figure 3: long term effects of TBI on mortality, climbing and sleep architecture

A) Kaplan-Meier estimates of survival functions in TBI treated flies and sham treated controls.

TBI (5 strikes) was induced in male w1118 flies $(n=59)$ and post-TBI survival was compared to survival in sham treated controls ( $n=57)$ using a log rank test. TBI results in a significant

311 decrease in survival rate $(p<0.001) .50 \%$ of the TBI group was deceased 14 days after TBI induction while $50 \%$ of the sham-treated controls had died 34 days after the start of the survival assay. B) Climbing behavior was tested in male w1118 flies., after which TBI was induced ( $n=$

314 30). Climbing behavior was subsequently tested for seven days after TBI and compared to sham-treated controls $(n=30)$. Climbing impairments recover on post-TBI days 2 and 3 , followed by a relapse on days 4-7 C) Sleep architecture was quantified in male flies up to ten

317 days after TBI induction $(n=103)$ and sham treated controls $(n=85)$. TBI induction resulted in 318 C) decreased total sleep for up to three days post TBI, D,E) more fragmented sleep (decreased 319 bout length, increased bout number) and E) increased brief awakenings, suggesting lighter 320 sleep. ${ }^{* * *} p<0.001,{ }^{* *} p<0.01$ by t-tests with Bonferroni correction). Errorbars indicate SEM. 
321 and sham-treated controls. Controls showed, on average, two to four TUNEL positive cells

322 which may be spontaneous apoptotic cells (Fig. 2C,D). Four hours after TBI induction we saw

323 an increase in TUNEL positive cells in the TBIx10 condition $\left(p=2.56 \times 10^{-6}\right)$, but not in the

324 TBIx5 condition $(p=0.1027 ; F(2,23)=68.29)$ at this time point (Fig. 2D). Eight hours after TBI

325 induction we also saw an increase in TUNEL positive cells in the TBIx10 condition $(p=2.93 \times$

$\left.32610^{-6}\right)$, but not in the TBIx5 condition $(p=0.5623 ; F(2,22)=33.41)$ at this time point (Fig. 2D). 24

327 hours after TBI induction we saw an increase in TUNEL positive cells in both the TBIx5 ( $p=$

$\left.3282.57 \times 10^{-6}\right)$ and the TBIx10 condition $\left(p=2.53 \times 10^{-6} ; F(2,19)=111.23\right)$ at this time point (Fig.

329 2D). ANOVA with Dunnett's post hoc test. Taken together, dCHI induces advanced mortality,

330 motor deficits and cell death within the first 24 hours.

$\underline{\mathrm{dCH}}$ reduces lifespan

333 Given the slowly evolving nature of TBI pathology, we next examined the chronic effects of

$334 \mathrm{dCHI}$ over time. We first examined lifespan. Unlike other forms of trauma, death after TBI rarely 335 occurs immediately. To test how our TBI assay affects overall lifespan, we delivered five 336 consecutive strikes to the top of a fly's head (Sup Movie 1). After this, flies were housed

337 individually and survivors were counted every day. $\mathrm{dCH}$ significantly reduces lifespan (log rank 338 test on Kaplan-Meier survival curves, $\mathrm{p}<0.001) .50 \%$ of the TBI group had died 14 days after 339 TBI induction while $50 \%$ of the sham-treated controls had died 32 days after the start of the 340 survival assay (Fig. 3A).

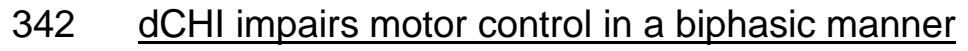

343 One day after being subjected to five consecutive hits, injured flies display a decrease in

344 climbing capacity, compared to sham-treated controls (Fig. 3B, p $<0.001$, t-test with Bonferroni

345 correction). Flies recover from days 2 to 3 , suggesting that climbing deficits are not due to

346 permanent injuries to the legs or to Johnston's organ, the fly's gravity sensor [68, 69]. 
347 Subsequently, flies undergo a relapse as climbing behavior is impaired again on days 4-7 ( $p<$

348 0.01, t-tests with Bonferroni correction). The biphasic response to $\mathrm{dCHI}$ mirrors a similar

349 biphasic motor response to TBI in a rodent model of TBI where rotarod performance was

350 decreased at 2 and 30 days post TBI, but not at 7 days post TBI [70].

$\underline{\mathrm{dCH} I \text { reduces and fragments sleep }}$

353 Sleep-wake disturbances after TBI are highly prevalent, occurring in $30-70 \%$ of TBI patients,

354 and consisting of insomnia, hypersomnia, fragmented sleep and altered sleep architecture

355 (reviewed in [71]). In rodent models of TBI, the most commonly reported sleep phenotypes are

356 increased total sleep [72] [73-76] and increased sleep fragmentation [72, 73, 75, 77-79].

358 To test whether sleep is impaired in our TBI model, flies were individually loaded into Drosophila

359 Activity Monitors immediately after TBI induction (5 strikes). The first three days post-TBI, sleep

360 was reduced in TBI treated flies compared to sham treated controls $(p<0.001$, t-test with

361 Bonferroni correction, Fig. 3C). Also, sleep was fragmented, as seen by a decrease in the

362 length of an average sleep bout $(p<0.001$, t-test with Bonferroni correction, Fig. 3D) and an

363 increase in the total number of sleep bouts ( $p<0.01$, t-test with Bonferroni correction, Fig. 3E).

364 Brief awakenings, a measure of sleep depth [45] were increased $(p<0.001$, t-test with

365 Bonferroni correction, Fig. 3F). These sleep phenotypes were not evident after 4 days (Fig. 3C-

366 F). Together, these results show that sleep in flies is decreased and fragmented in the first few

367 days after TBI, but that it returns to baseline after four days.

$369 \quad \mathrm{dCHI}$ acutely activates the innate immune response

370 To test whether $\mathrm{dCHI}$ could alter glial gene expression, we used Translating Ribosome Affinity

371 Purification and Sequencing (TRAP-seq). TRAP-seq allows for a cell type-specific analysis of

372 the all mRNAs that ribosome associated and thus, are potentially being translated $[48,80]$. 
A

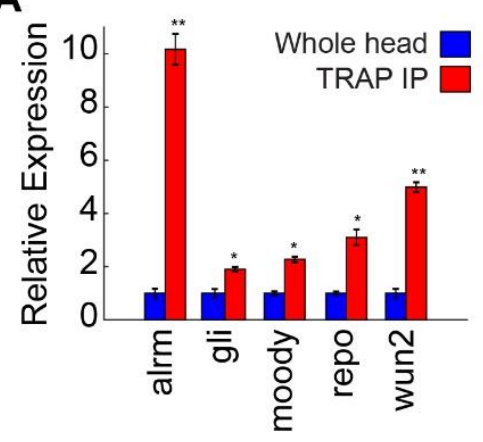

B

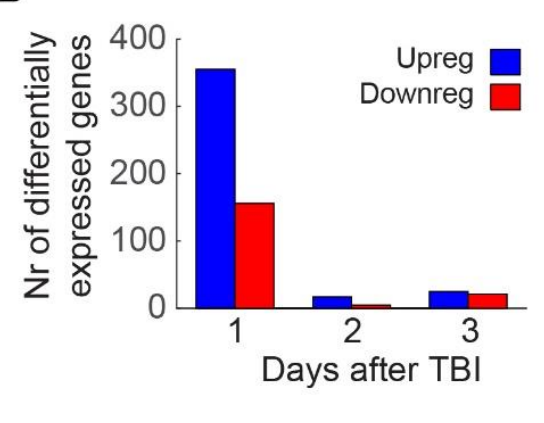

C

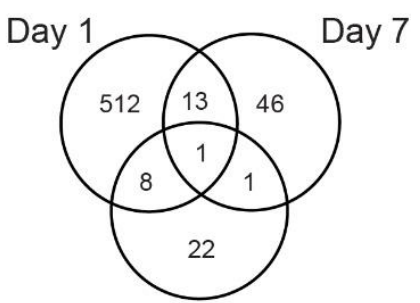

Day 3

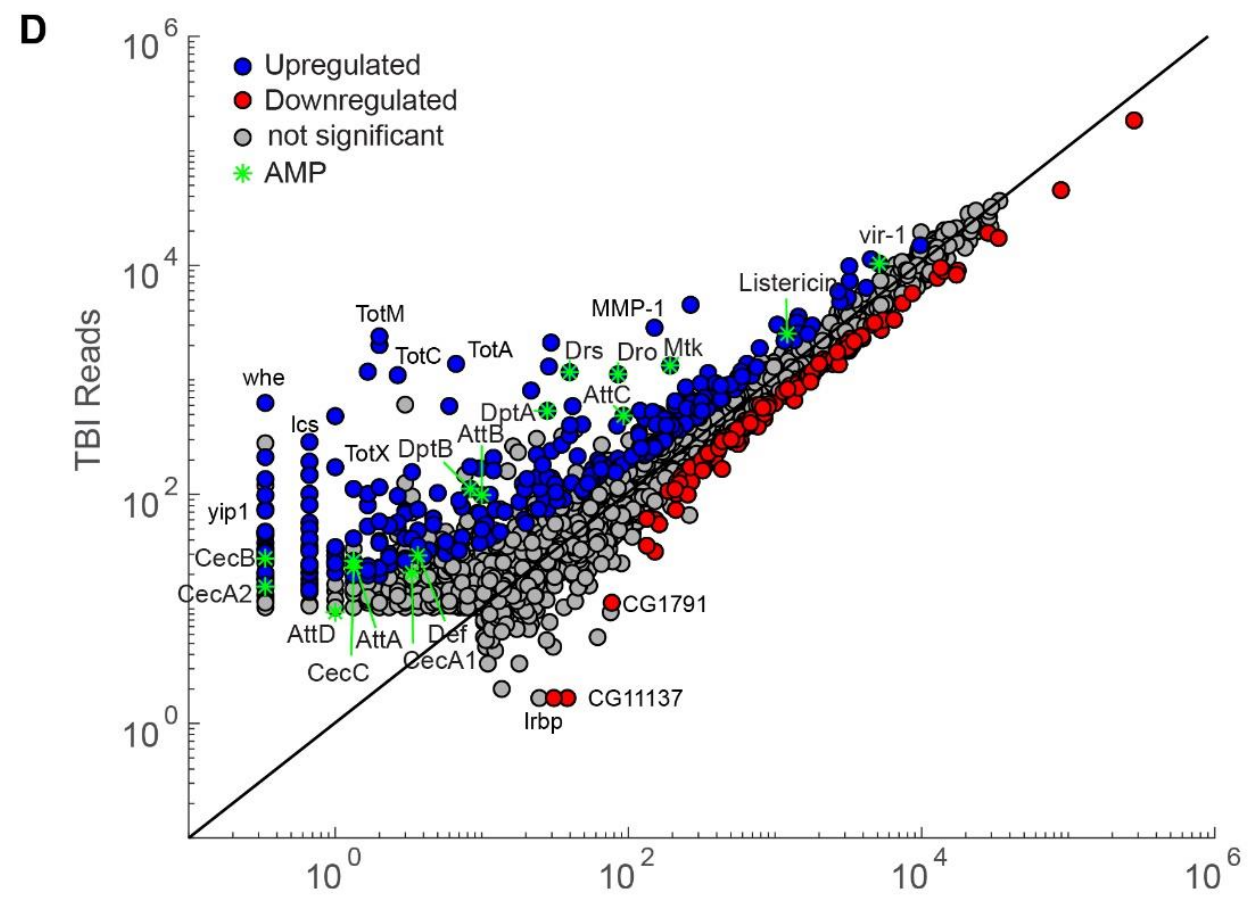

Control Reads

\section{Figure 4 - TBI causes changes in gene expression}

A) Expression levels for whole head inputs are compared to glia-specific TRAP

immunoprecipitated (TRAP IP) and show that glia-specific genes are enriched, including astrocytic leucine-rich repeat molecule (alrm, astrocyte-specific), gliotactin (gli, expressed in

378 peripheral glia), moody (blood brain barrier), reversed polarity (repo, pan-glial) and wunen-2

379 (wun2, astrocyte-specific). Data is normalized for whole head inputs (3 replicates for both whole

380 head inputs and TRAP IP, ${ }^{* *} p<0.01,{ }^{*} p<0.05, t$-test). Errorbars show SEM. B) TBI induction

381 results in a large number of differentially expressed genes in glia cells. 356 genes are

382 upregulated and 156 are downregulated 1 day after TBI induction. Three days after TBI, gene 
expression is almost back to baseline, with only 17 genes that are upregulated and 5 genes that are downregulated. Seven days after TBI differential gene expression 24 genes are upregulated and 22 are downregulated. C) Venn diagram showing the overlap of differentially expressed genes (upregulated + downregulated) on 1, 3 and 7 days after TBI induction. D) Scatter plot for glial genes where average reads in the control condition are plotted against average reads 24 hours after TBI (blue dots, $\log _{2}$ fold change $\geq 0.6$, Benjamini adjusted $p<0.1$ ) or downregulated (red dots, $\log _{2}$ fold change $\leq-0.6$, Benjamini adjusted $\left.p<0.1\right) 24$ hours after TBI induction. AMPs are indicated with green asterisks. Genes with average reads $<10$ in both control and TBI condition were excluded.

We used the pan-glial driver repo-GAL4 [81] to drive UAS-GFP::RpL10A, a GFP-tagged version

394 of a ribosomal protein [48]. Head RNA was isolated at 1, 3 and 7 days after TBI induction and compared them to sham-treated controls. Ribosome associated mRNAs were isolated by immunoprecipiation of the RpL10A-GFP. For each time point, three replicates $(n=\sim 200$ male fly heads/replicate) were collected. Gene expression levels were determined using Kallisto-derived estimated counts of glial TRAP-seq data collected at 1,3, or 7 days after dCHI. Due to technical

399 issues with one of day 7 TBI replicates, it was removed from further analysis (see Methods and 400 Fig. S1).

402 To validate the method, we assessed enrichment of known glial genes in the anti-GFP 403 immunoprecipitate (IP) relative to the input of whole head mRNAs (Fig. 4A). Expression levels

404 for non-glia inputs were compared to glia-positive controls and show that glia-specific genes are 405 enriched, including astrocytic leucine-rich repeat molecule (alrm)[29]) and wunen-2 (wun2)[82], 406 which are expressed highly in astrocytes, gliotactin, a transmembrane protein on peripheral glia 
[83]), moody (GPRCs that regulate blood brain barrier permeability [84]) and reversed polarity (repo) [81].

409

410 We initially looked at genes that are differentially expressed between $\mathrm{dCH}$ and sham-treated

411 controls. $\log _{2}$ fold change shows how gene expression levels change after $\mathrm{dCHI}$, compared to

412 sham-treated controls. P-values are adjusted using the Benjamini-Hochberg procedure to

413 correct for false discovery rates [85]. We first noted that the number of differentially expressed

414 genes on day 1 post-dCHI vastly outnumber those evident on day 3 or day 7 . In general, the

415 number of upregulated genes is higher than the number of downregulated genes (Fig. 4B, Fig.

416 S2). The differentially regulated genes are qualitatively different as well, with little overlap

417 among differentially expressed genes on post-TBI day 1, 3 and 7 (Fig. 4C).

419 By plotting the average gene levels in the control group against those in TBI-treated flies (Fig.

420 4D), we see that TBI results in genes being more strongly upregulated than downregulated

421 (more blue dots, further away from unity line). Strongly upregulated genes include antimicrobial

422 peptides (AMPs) (Drosomycin (Drs), listericin, virus-Induced RNA-1 (vir-1), genes involved in

423 proteolysis (alphaTry, yip7), and autophagy (MMP1). Also, quite a few members of the Turandot

424 (Tot) family are upregulated after TBI (TotA, TotC, TotM, TotX). These genes are part of a broad

425 stress response in Drosophila and are upregulated after exposure to mechanical stress, heat,

$426 \mathrm{UV}$, bacteria, oxidative stress and dehydration [86]. Turandot $A(\operatorname{Tot} A)$ is strongly induced by

427 bacterial challenge as well as exposure to mechanical pressure, dehydration and oxidative

428 stress. [86]. We also find that several genes with poorly understood functions are also strongly

429 upregulated after TBI (whatever (whe), la costa (Ics)). 


\begin{tabular}{|c|c|c|c|c|}
\hline Term & Count & $\%$ & P-Value & Benjamini \\
\hline proteolysis & 55 & 10.8 & $6.40 \mathrm{E}-14$ & $5.70 \mathrm{E}-11$ \\
\hline antibacterial humoral response & 13 & 2.6 & 4.70E-12 & $2.10 \mathrm{E}-09$ \\
\hline defense response to Gram-positive bacterium & 18 & 3.5 & 7.30E-12 & 2.20E-09 \\
\hline response to bacterium & 16 & 3.1 & $4.00 \mathrm{E}-11$ & $9.00 \mathrm{E}-09$ \\
\hline innate immune response & 22 & 4.3 & 1.30E-10 & $2.30 \mathrm{E}-08$ \\
\hline defense response & 11 & 2.2 & 2.30E-07 & $3.50 E-05$ \\
\hline humoral immune response & 9 & 1.8 & $1.30 \mathrm{E}-06$ & $1.70 \mathrm{E}-04$ \\
\hline protein folding & 16 & 3.1 & $8.60 \mathrm{E}-06$ & $9.60 \mathrm{E}-04$ \\
\hline response to heat & 12 & 2.4 & $3.30 E-05$ & $3.20 \mathrm{E}-03$ \\
\hline defense response to Gram-negative bacterium & 15 & 3 & $3.80 \mathrm{E}-05$ & $3.40 E-03$ \\
\hline defense response to bacterium & 11 & 2.2 & $1.50 \mathrm{E}-04$ & $1.20 E-02$ \\
\hline protein refolding & 5 & 1 & $3.10 \mathrm{E}-04$ & $2.30 \mathrm{E}-02$ \\
\hline rRNA pseudouridine synthesis & 4 & 0.8 & 4.60E-04 & $3.10 \mathrm{E}-02$ \\
\hline response to methotrexate & 5 & 1 & $7.00 \mathrm{E}-04$ & 4.30E-02 \\
\hline glutathione metabolic process & 8 & 1.6 & $1.50 \mathrm{E}-03$ & $8.50 E-02$ \\
\hline dorsal trunk growth, open tracheal system & 4 & 0.8 & $1.50 \mathrm{E}-03$ & $8.20 \mathrm{E}-02$ \\
\hline cellular response to oxidative stress & 6 & 1.2 & $1.50 \mathrm{E}-03$ & $7.80 \mathrm{E}-02$ \\
\hline chaperone-mediated protein folding & 5 & 1 & 1.80E-03 & $8.30 \mathrm{E}-02$ \\
\hline cytoskeleton organization & 7 & 1.4 & $1.80 \mathrm{E}-03$ & $8.00 \mathrm{E}-02$ \\
\hline response to oxidative stress & 11 & 2.2 & $1.90 \mathrm{E}-03$ & $8.30 \mathrm{E}-02$ \\
\hline cell adhesion & 11 & 2.2 & 2.30E-03 & $9.20 \mathrm{E}-02$ \\
\hline transmembrane transport & 22 & 4.3 & 2.30E-03 & $8.90 \mathrm{E}-02$ \\
\hline
\end{tabular}

Immune response (115/286)

Protein folding/breakdown (85/286)

Physiological stress response (42/286)

\section{Other}

434 Table 1: Enriched biological processes 24 hours after TBI

436 as well as several physiological stress responses and proteolytic cascades. 
440 The biological processes that are elevated 24 hours after TBI can be roughly grouped in three

441 different categories: immune-related, proteolytic/protein folding and stress response processes.

442 The majority of processes are part of the immune response (Table 1, red), including innate

443 immune responses, humoral immune responses and different classes of antimicrobial peptides

444 (AMPs). Drosophila AMPs can be grouped into three families based on their main biological

445 targets, gram-positive bacteria (Defensin), gram-negative bacteria (Cecropins, Drosocin,

446 Attacins, Diptericin), or fungi (Drosomycin, Metchnikowin) [87]. Most AMP genes were shown to

447 be present in a TRAP-seq analysis of Drosophila astrocytes, a glial subset [88]. dCHI results in

448 increased differential expression of many AMPs, including Attacins, Cecropins and Diptericins

449 as well as Drosocin, Drosomycin and Metchnikowin (Fig. 5, blue bars). These AMPs are

450 regulated by the Toll, Imd and JAK-STAT pathways [89]. Previous Drosophila TBI models

451 showed an increase in three antimicrobial peptides (AMPs) after TBI induction, Attacin-C,

452 Diptericin B and Metchnikowin [40, 41], but due to the non-specific nature of the impact, it is

453 uncertain whether they are caused by TBI or by other types of injury. Enriched products of the

454 antiviral and antibacterial JAK-STAT cascade are Listericin - an antibacterial protein and Virus-

455 induced RNA 1, a marker of the induction of an antiviral response. In Drosophila, many

456 proteases are involved in the immune response, including the activation of the Toll ligand

457 Spätzle, which is under the control of a serine protease cascade [90]. These proteolytic

458 cascades play a crucial role in innate immune reactions because they can be triggered more

459 quickly than immune responses that require altered gene expression [91]. Expression of serine

460 proteases that regulate Toll activation (Spirit, Grass, SME, Spheroid, Sphinx 1/2 [92]) were not

461 significantly altered in our assay (data not shown). However, Späztle, a ligand for the Toll

462 pathway that binds to Toll to activate the pathway [93], was upregulated. Activation of the

463 immune response gradually dies down, as only three AMPs are upregulated three days after

464 TBI. Seven days after TBI, all AMPs have returned to baseline levels (Fig. 5). 


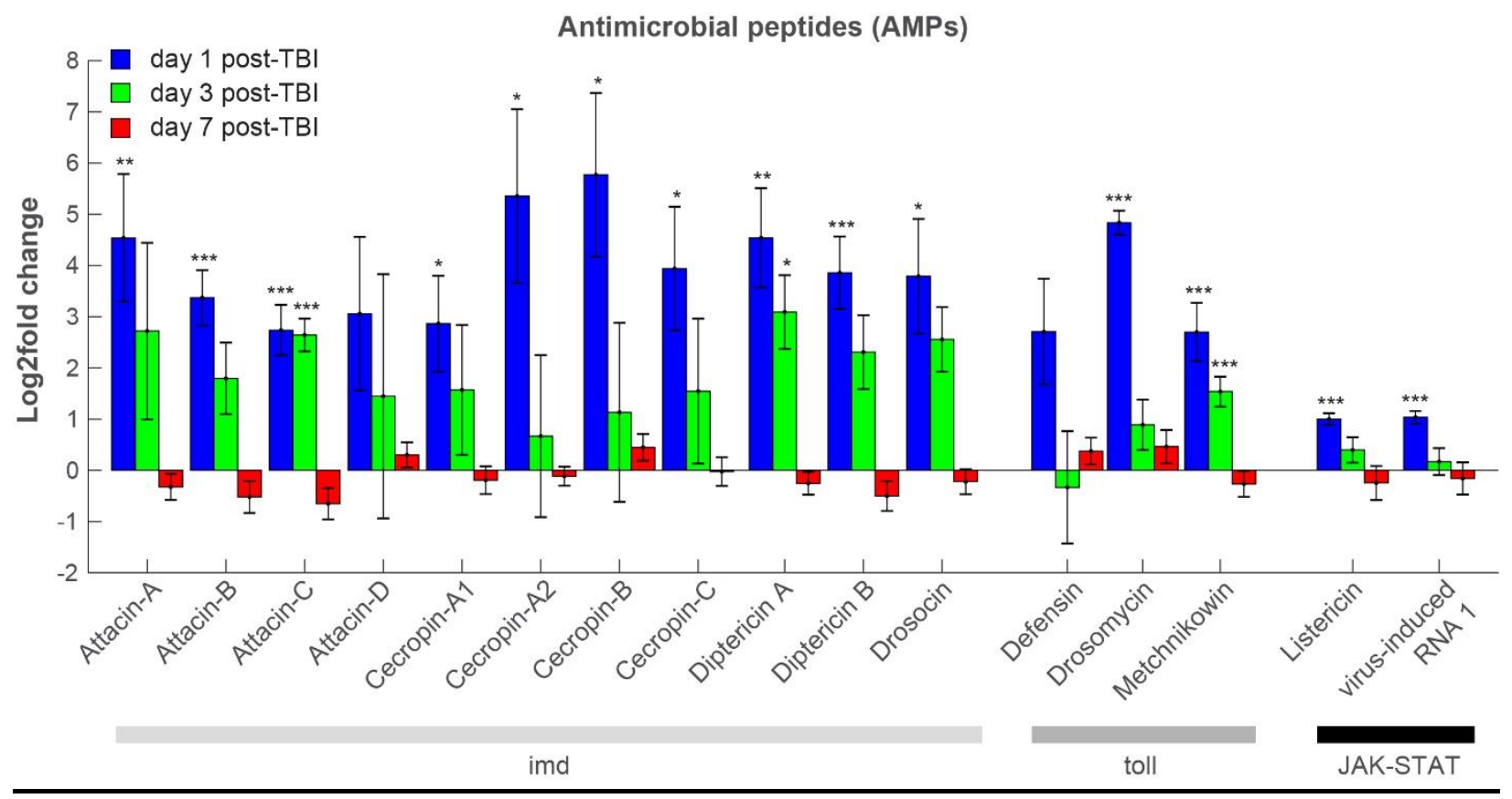

467 Figure 5 - TBI activates a broad innate immune response

24 hours after TBI, genes encoding most Drosophila antimicrobial peptides (AMPs) are enriched in glia cells. These include antibacterial, antifungal and antiviral peptides that are regulated by the Toll, Imd and JAK-STAT pathways. Three days after TBI (green), all but three AMPs (AttC, $D p t A$, Mtk) have returned to control levels. Seven days after TBI (red) no AMPs are up- or down-regulated. Gene expression levels are considered to have changed significantly if the log2fold change $\geq|0.6|$ and the Benjamini-adjusted $p$-value is $<0.1{ }^{* * *}$ adj $p<0.001,{ }^{* *}$ adj $p<$ $0.01,{ }^{*}$ adj $p<0.1$. Errorsbars indicate SEM.

In addition to immune gene activation, transcriptomics also uncovered novel pathways that

477 mediate TBI effects. A second category of enriched biological processes after TBI are proteases (Table 1). For example, we detected strong upregulation of matrix metalloproteinase-

4791 (MMP-1, $1.79 \log _{2}$ fold change, adj $\left.p=4.62 E-23\right)$. MMPs are a family of endopeptidases that 480 have diverse physiological and pathological functions, including degradation of extracellular 
ensheathing glia responding to axonal injury and is required for glial clearance of severed axons

[35]. Detecting MMP-1 in our $\mathrm{dCHI}$ assay is further evidence that $\mathrm{TBI}$ damages axons and

484 triggers glia-mediated neuroprotective responses.

The third main category of enriched genes 24 hours after TBI are stress response genes, genes

487 that are upregulated in response to a variety of stressful stimuli, including heat, oxidative,

488 metabolic and chemical stress [86, 95] (Table 1, green), including the Tot genes and heat shock

489 proteins $22,23,26,27,68,70 \mathrm{Bc}$ and lethal-2 as well as genes involved in oxidative stress. Heat

490 shock protein 27 was previously implicated in response to bacteria and fungi as well [96].

492 In our model, TBI causes sleep to be reduced, fragmented and less deep (Fig.3C-F), a

493 phenotype that persists for three days after TBI induction. Two prominent sleep regulating

494 genes have altered expression levels 24 hours after TBI. Dopamine transporter (DAT, also

495 known as fumin), a dopamine transporter that mediates uptake of dopamine from the synaptic

496 cleft, is downregulated $\left(-0.74 \log _{2}\right.$ fold change, $\left.\mathrm{p}=0.021\right)$. Loss of $D A T$ increases extracellular

497 dopamine and is associated with increased activity and decreased sleep [97-99]. Pale (ple), a

498 tyrosine hydroxylase that drives synthesis of wake-promoting dopamine [68], is upregulated

499 (0.63 $\log _{2}$ fold change, $\left.p=0.018\right)$. Pale was previously shown to be activated in response to

500 wounding in Drosophila embryos and larvae [100]. Pale and DAT/fumin levels are not changed

501 on post-TBI days 3 and 7 (data not shown). Thus, we hypothesize down regulation of

502 DAT/fumin in combination with upregulation of pale may underlie TBI changes to sleep due to

503 increased dopamine levels.

504

505 Three days after TBI induction, there are few significant differences in gene expression between

506 TBI treated flies and sham treated controls (Fig. 4B, Fig. S3A). Whereas there are 512 genes

507 with altered expression 24 hours post-TBI (almost 400 of those are upregulated), after three 
508 days there are only 22 genes with altered expression levels (Fig. 4B). Interestingly, this low level

509 of glial activation at day 3 post-TBI coincides with a climbing behavior returning back to control

510 levels (Fig. 4B). At three days post TBI, several AMPs remain strongly upregulated (AttC, Mtk,

511 Dpt; Fig. 5, Fig. S3A).

512

513 Seven days after TBI induction, there is more variability in gene expression (Fig. S1E,F). There

514 seems to no activation of the immune response, as all AMPs have returned to baseline levels

515 (Fig. 5, red bars). There is only one gene that is persistently downregulated on days 1, 3 and 7

516 (Fig. 4C). CG40470's function is unknown, although roles in proteolysis and peptide catabolic

517 processes have been inferred [101].

519 Post-TBI behavioral phenotypes are NF-kB -dependent

520 The NF-kB family of transcription factors plays a central role in the regulation of inflammatory

521 gene expression [102], cell survival and neuronal plasticity [103]. NF-kB is activated in neurons

522 and glial cells after injury and has been linked to both neurodegenerative and neuroprotective

523 activities [103, 104]. NF-kB mediates activation of glial cells [105] and inflammation [106]. TBI

524 causes an increase in Nuclear Factor kappa B (NF-kB) in rodents [107-109], where it has a

525 neuroprotective effect in a closed-head model of TBI [70]. In Drosophila, NF-kB is a crucial

526 component of both the Toll and the Imd pathways, where different isoforms are required for the

527 expression of different antimicrobial peptides [110]. Changes to sleep architecture after

528 injury/infection in Drosophila require NF-KB Relish [111]. In Drosophila, over-expression of NF-

$529 \mathrm{~KB}$ or AMPs in glia cells causes neurodegeneration [38, 39, 112, 113]. 
A

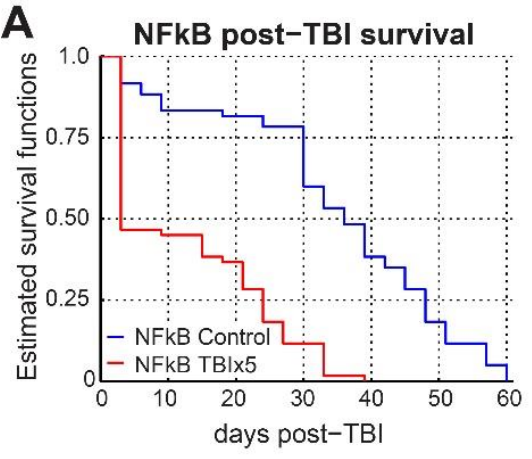

D

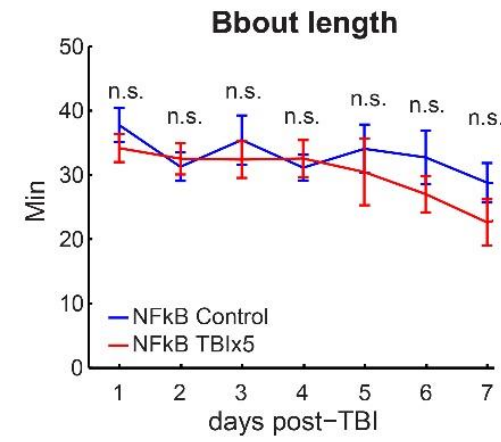

B

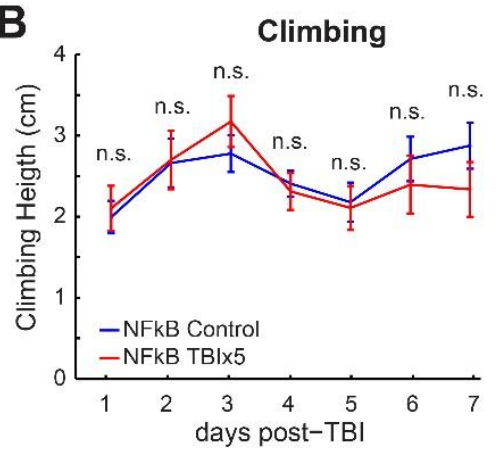

E

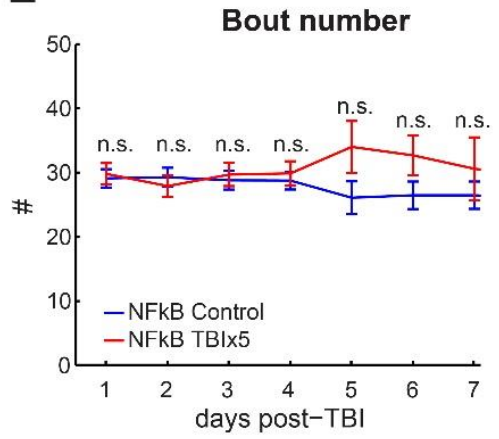

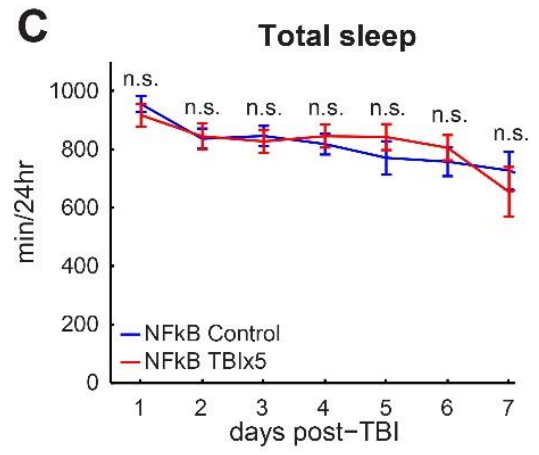

$\mathbf{F}$

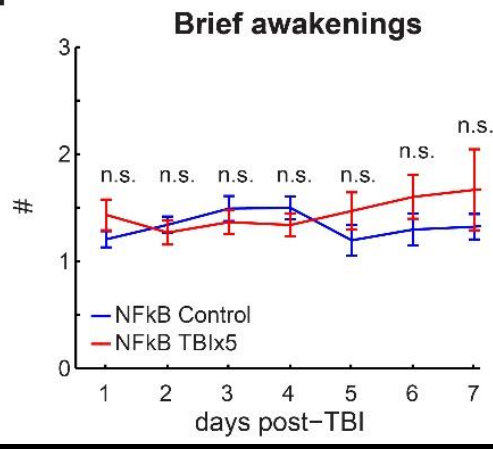

\section{Fig 6 - Post-TBI behavioral phenotypes are NF-kB dependent}

533

534

535

536

537

538

539

540

541

542

543

544

A) Kaplan-Meier estimates of survival functions in TBI treated NF-KB null mutants and sham treated controls ( $n=60 /$ group) show that TBI significantly reduces lifespan (log rank test, $p$ $<0.001)$. B) The effect of TBI on climbing behavior was tested in male NF-KB null mutants and sham-treated controls ( $n=32 /$ group for seven consecutive days after TBI induction. There was no significant difference between both groups (n.s., t-tests with Bonferroni correction. C) TBI does not impair sleep in NFKB null mutants, compared to sham-treated controls. TBI does not fragment sleep as average bout length $(\boldsymbol{D})$ and bout number $(\boldsymbol{E})$ are not changed. $\boldsymbol{F})$ Brief awakenings, a measure of sleep depth, are unchanged after TBI. ( $n=52$ controls, 57 in TBI group, n.s.; t-tests with Bonferroni correction). Errorbars indicate SEM. 
545 To test the role of NF-KB in TBI-induced mortality and behavioral impairments, we induced TBI

546 in the NF-kB Relish null mutant (w1118; Rel[E20], Bloomington \#9457) and measured its effects

547 on post-TBI survival, climbing behavior and sleep. Five consecutive strikes to the head resulted

548 in strongly increased mortality (log rank test on Kaplan-Meier survival curves, $p<0.001$ ), where

549 over $50 \%$ of the NF-kB mutants had died three days after TBI induction (Fig. $6 \mathrm{~A}$ ). In the

550 background strain $\left(w^{1118}\right), 50 \%$ mortality for five strikes occurs at $\sim 14$ days post-TBI (Fig.3A). In

551 sham-treated NF-KB controls, 50\% mortality occurs after 35 days (Fig. 6A), which is very similar

552 to mortality in the sham-treated $w^{1118}$ background strain (Fig. 3A).

554 To test whether TBI has a much stronger effect on NF-kB mutants, we tested climbing behavior

555 daily for seven days after TBI induction. However, there was no difference between TBI treated

556 flies and untreated controls on any day (Fig. 6B). This is different from wild-type flies which

557 show considerable impairment to climbing 24 hours post-TBI, which then reverts back to normal

558 and is followed by a relapse on days 5-7 (Fig. 3B). Both TBI treated and sham treated controls

559 show a gradual decrease in total sleep over ten days post-treatment. However, there are no

560 differences in total sleep (Fig. 6C) or in any other metrics of sleep architecture (brief

561 awakenings, bout length, bout number, wake activity; Fig. 6D-F). These results suggest that the

562 NF-kB-dependent immune response facilitates survival after TBI but that impairments in sleep

563 and climbing behavior are consequences of an immune-dependent injury mechanism.

\section{Discussion}

566 Here we have developed a simple and reproducible Drosophila model for closed head TBI

567 where we deliver precisely controlled strikes to the head of individually restrained,

568 unanesthetized flies. This TBI paradigm is validated by recapitulating many of the phenotypes

569 observed in mammalian TBI models, including increased mortality, increased neuronal cell

570 death, impaired motor control, decreased/fragmented sleep and hundreds of TBI-induced 
571 changes to the transcriptome, including the activation of many antimicrobial peptides, indicating

572 a strong activation of the immune response. Behavioral responses to TBI (e.g. sleep and

573 geotaxis) are abolished in mutants of the transcription factor NF-kB Relish, which plays a central

574 role in regulating stress-associated and inflammatory gene expression in both mammals [103,

$575114]$ and flies [115]. Nonetheless, Relish null mutants show increased mortality after TBI. These

576 results set the stage to leverage Drosophila genetic tools to investigate the role of the immune

577 response as well as novel pathways in TBI pathology.

579 Our single fly paradigm is a more valid Drosophila model for TBI that circumvents the lack of

580 specificity of currently available models [39, 41]. Both previous assays induce TBI by subjecting

581 the whole fly to trauma, which makes it hard to distinguish whether observed phenotypes are a

582 due to traumatic brain injury or a consequence of internal injuries. A recently published method

583 [116] uses a pneumatic device to strike an anesthetized fly's head. This method is an

584 improvement of earlier assays and results in increased mortality in a stimulus strength

585 dependent manner. However, it only shows a modest reduction in locomotor activity, without

586 demonstrating any other TBI-related phenotypes such as neuronal cell death or immune

587 activation. The dependence on $\mathrm{CO}_{2}$ anesthesia further impairs the usefulness of this assay, as

588 prolonged behavioral impairments in Drosophila occur even after brief exposure to $\mathrm{CO}_{2}$

589 anesthesia [117]. Additionally, anesthetics that are administered either during or shortly after

590 TBI induction can offer neuroprotective effects and alter cognitive, motor, and histological

591 outcomes in mammalian models of TBI [118-120] as well as affecting mortality in a whole-body

592 injury model in flies [121]. Our Drosophila model allows us to study how TBI affects behavior

593 and gene expression without the confounding effects of anesthesia, making it a more valid

594 model for TBI that occurs under natural conditions. 
596 In this study, we also elucidate in an unbiased manner, the genomic response to TBI. Glial cells

597 play an important role in immune responses in both mammals and Drosophila, (reviewed in

598 [33]). Until now, profiling TBI-induced changes in gene expression have either been limited to a

599 small number of pre-selected genes in both mammals [122, 123] and Drosophila [40, 41] or

600 focused on whole brain tissue rather than individual cell types [124, 125]. Using TRAP in

601 combination with RNA-seq, we detect an acute, broad-spectrum immune response, where

602 antimicrobial peptides (AMPs) and stress response genes are upregulated 24 hours after TBI.

603 These include antibacterial, antifungal and antiviral peptides as well as peptides from the

604 Turandot family which are secreted as part of a stress response induced by bacteria, UV, heat

605 and mechanical stress [86]. Three days after TBI, only Attacin-C, Diptericin A and Metchnikowin

606 are upregulated. Seven days after TBI, AMPs or stress response genes are not detectably

607 upregulated. These findings match reports in mammalian TBI models, where inflammatory gene

608 expression spikes shortly after TBI but mostly dies down during subsequent days [122, 126].

610 Besides validating our Drosophila model with the detection of a strongly upregulated immune

611 response, we detected several novel genes among the total of 512 different glial genes that

612 were either up- or down-regulated after TBI. Immune and stress response only make up 157 out

613 of 512 differentially expressed glial genes. Genes involved in proteolysis and protein folding are

614 a prominent portion (85/512) of these differentially expressed genes, yet their role in TBI is

615 poorly understood. These results demonstrate that there are other candidate pathways that may

616 modulate recovery, and Drosophila can be used as a first line screen to test their in vivo

617 function.

618

619 We have successfully applied in vivo genetics to identify in vivo pathways important for TBI.

620 Here we demonstrate that loss of master immune regulator NF-KB results in increased mortality

621 after TBI, yet it protects against TBI-induced impairments in sleep and motor control. These 
622 findings align with previous reports showing links between sleep and the immune response in

623 flies [127] where NF-kB is required to alter sleep architecture after exposure to septic or aseptic

624 injuries [128]. One possibility is that sleep impairments can be a side-effect of melanization, an

625 invertebrate defense mechanism that requires dopamine as melanin precursor [129]. If

626 dopamine is upregulated to create more melanin, decreased sleep would be a side effect.

627 Consistent with this hypothesis, we observe changes in fumin and pale which could results in

628 increased dopamine levels. Recently, it was shown that repressing neuronal NF-KB in a mouse

629 model of TBI increases post-TBI mortality, as in our studies, without reducing behavioral

630 impairments [70], suggesting that non-neuronal NF-kB could underlie behavioral impairments

631 after TBI. The demonstration of an in vivo role for TBI-regulated genes will be important for

632 defining their function.

634 In summary, our Drosophila Closed Head Injury assay $(\mathrm{dCHI})$ recapitulates many of the

635 physiological symptoms observed in mammals, demonstrating that fruit flies are a valid model to

636 study physiological responses to TBI. We demonstrate both a potent induction of immune

637 pathways and a requirement for an immune master regulator in mediating TBI effects on

638 behavior. Our model now provides a platform to perform unbiased genetic screens to study how

639 gene expression changes after TBI in unanesthetized, awake animals result in the long-term

640 sequelae of TBI. These studies raise the possibility of rapidly identifying key genes and

641 pathways that are neuroprotective for TBI, thereby providing a high-throughput approach that

642 could facilitate the discovery of novel genes and therapeutics that offer better outcomes after

643 TBI. 

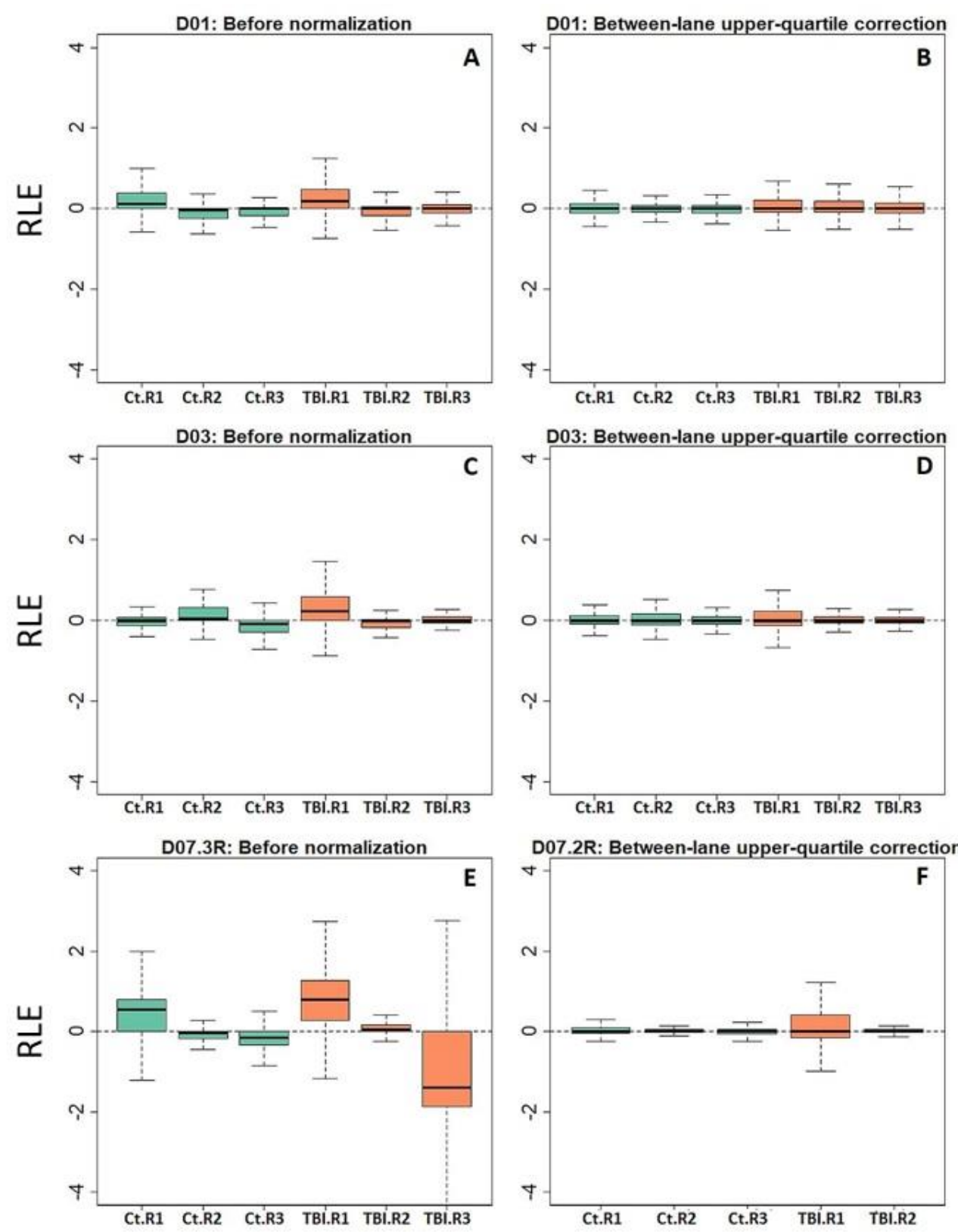

Fig S1 - Relative log expression and normalized data for post-TBI days 1, 3 and 7

651 Relative log expression (RLE) plot of raw and normalized glial expression data. Control (green)

652 and TBI (orange) biological replicates for day 1, 3 and 7 post-TBI. Correction was performed

653 using the upper-quartile between lane method. Due to the high variability in TBI replicate 3 on

654 day 7 , this replicate was discarded 


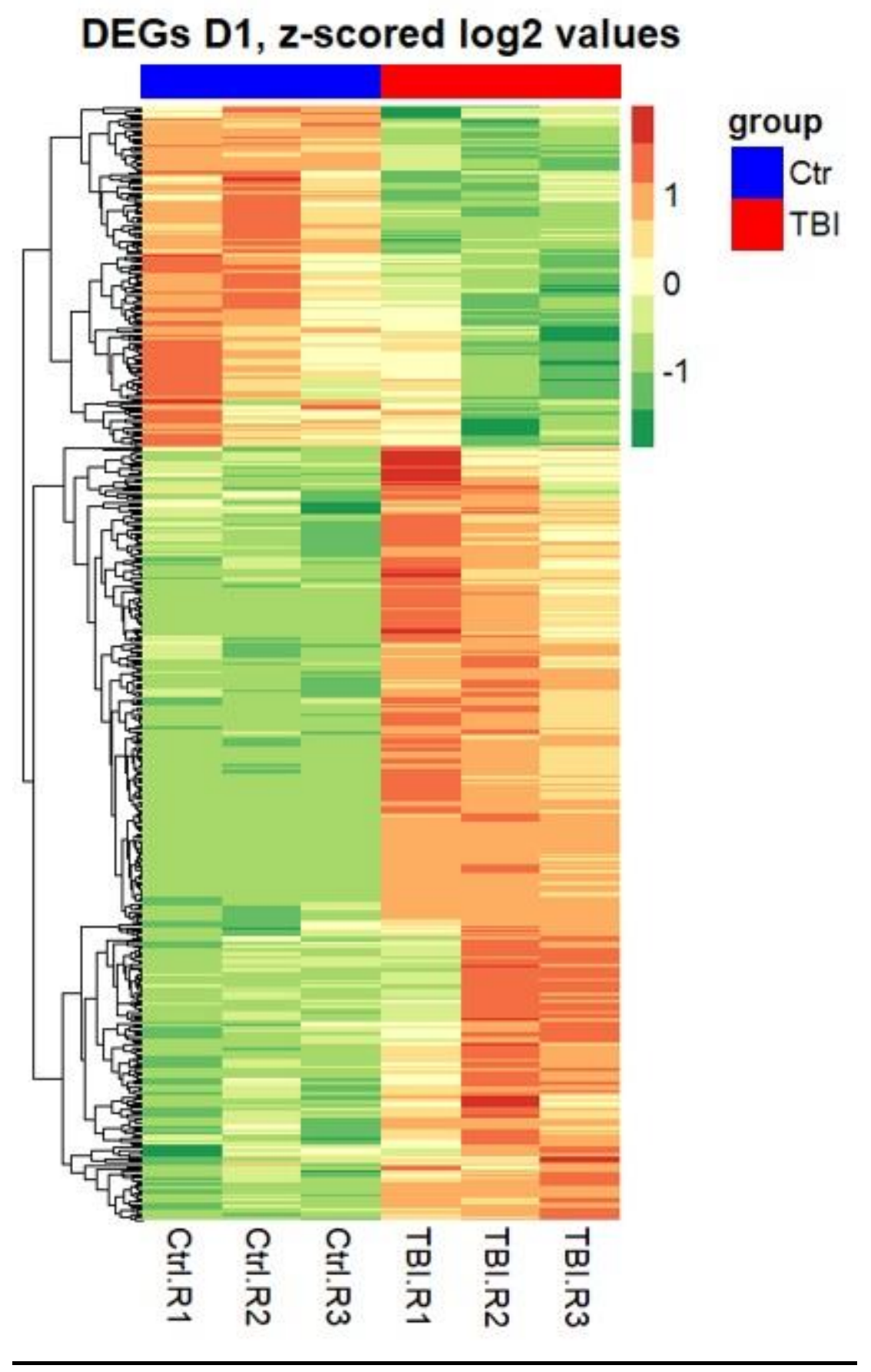

656 Fig S2: Glial gene expression heat map. Panels present clustering of differentially expressed

657 genes (DEGs) for day 1 post-TBI. Gene expression level presented as z-scored log2(X+1)

658 transformed values; Control replicates in blue, TBI replicates in red. 


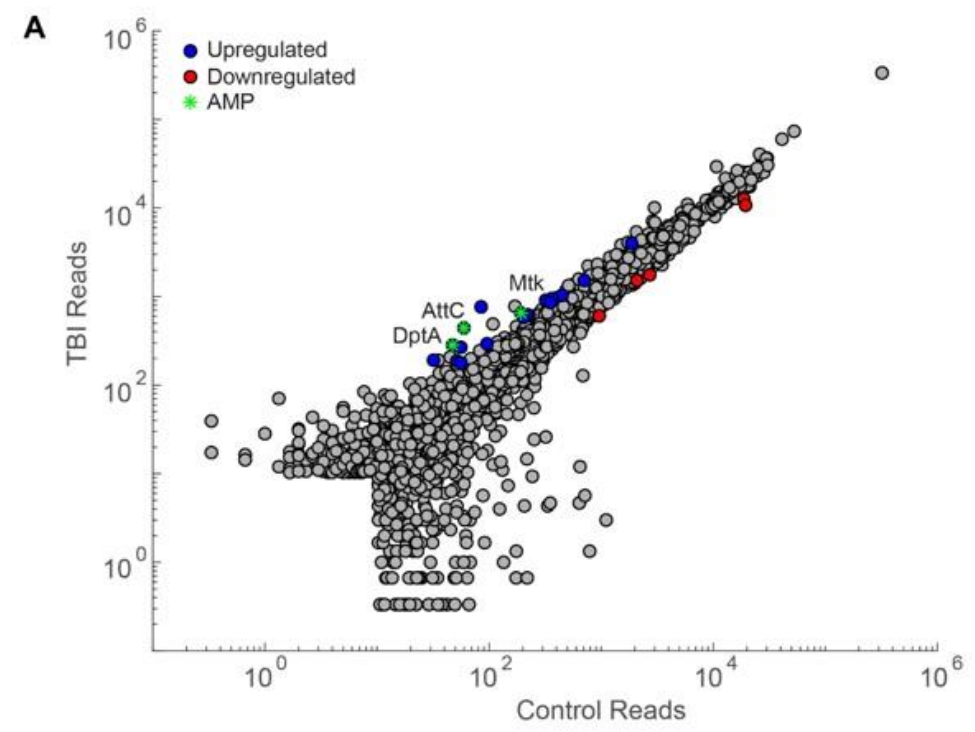

B

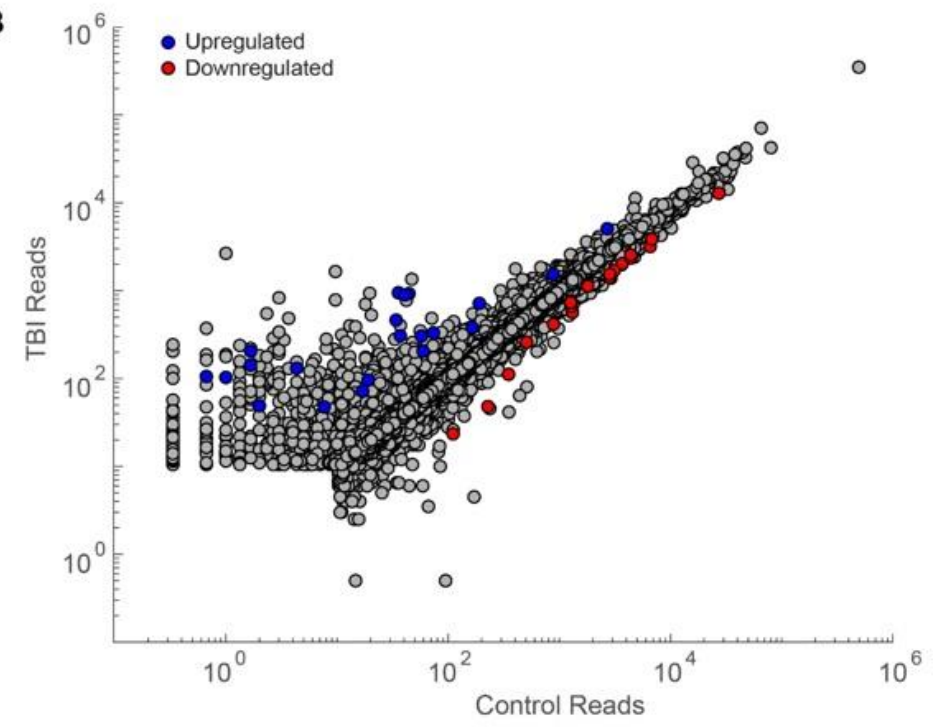

Figure S3 - Differentially expressed genes in repo-TRAP at 3 and 7 days post-TBI

662 Scatter plot for glial genes where average reads in the control condition are plotted against

663 average reads three days $(\mathbf{A})$ or seven days $(B)$ after TBI (blue dots, $\log _{2}$ fold change $\geq 0.6$,

664 Benjamini adjusted $p<0.1$ ) or downregulated (red dots, $\log _{2}$ fold change $\leq-0.6$, Benjamini

665 adjusted $p<0.1) 24$ hours after TBI induction. AMPs are indicated with green asterisks. Genes

666 with average reads $<10$ in both control and TBI condition were excluded. 
Author Information

668

669 B van Alphen \& S Stewart

670 These authors contributed equally

671

\section{Affiliations}

673 Department of Neurobiology, Northwestern University, Evanston, IL 60208, USA

674 B van Alphen, S Stewart, M Iwanaszko, F Xu, E Bang, S Rozenfeld, A Ramakrishnan, T.Q. Itoh 675 \& R Allada

676

677 Department of Preventive Medicine - Biostatistics, Feinberg School of Medicine, Northwestern

678 University, Chicago, 60611 IL, USA

679 M Iwanaszko \& R.I. Braun

680

681 Department of Engineering Sciences and Applied Mathematics, Northwestern University,

682 Chicago, 60208 IL, USA

683 R.I. Braun

684

\section{Author contributions}

686 BvA developed the TBI assay. Experiments were designed by BvA, SS and RA. Sleep, climbing 687 and mortality assays were performed by BvA and SS. TUNEL assays were performed by SR 688 and AR. NF-kB experiments were carried out by BvA and EB. TRAP seq was conducted by SS,

689 FX and TQI. RNA-preprocessing, differential gene expression and functional analysis were 690 performed by $\mathrm{MI}$ and RIB. BvA and RA wrote the manuscript. 


\section{Competing interests}

694 The authors declare no competing interests

695

696 Corresponding author

697 Correspondence to B van Alphen or R Allada

698

699 Code availability. The code used to generate the results that are reported in this study are

700 available from the corresponding author upon reasonable request.

701 Data availability. The data that support the findings of this study are available from the

702 corresponding author upon reasonable request

703

704

705

706

707

708

709

710

711

712

713

714

715

716

717

718 


\section{References}

720 1. Maas, A.I., N. Stocchetti, and R. Bullock, Moderate and severe traumatic brain injury in

adults. Lancet Neurol, 2008. 7(8): p. 728-41.

2. Menon, D.K., et al., Position statement: definition of traumatic brain injury. Arch Phys Med Rehabil, 2010. 91(11): p. 1637-40.

3. Langlois, J.A., W. Rutland-Brown, and M.M. Wald, The epidemiology and impact of traumatic brain injury: a brief overview. J Head Trauma Rehabil, 2006. 21(5): p. 375-8.

4. Sauaia, A., et al., Epidemiology of trauma deaths: a reassessment. J Trauma, 1995. 38(2): p. 185-93.

5. Gaetz, M., The neurophysiology of brain injury. Clin Neurophysiol, 2004. 115(1): p. 4-18.

6. Blennow, K., J. Hardy, and H. Zetterberg, The neuropathology and neurobiology of traumatic brain injury. Neuron, 2012. 76(5): p. 886-99.

7. Ransohoff, R.M. and B. Engelhardt, The anatomical and cellular basis of immune surveillance in the central nervous system. Nat Rev Immunol, 2012. 12(9): p. 623-35.

8. Loane, D.J. and K.R. Byrnes, Role of microglia in neurotrauma. Neurotherapeutics, 2010. 7(4): p. 366-77.

9. Morganti-Kossmann, M.C., et al., Role of cerebral inflammation after traumatic brain injury: a revisited concept. Shock, 2001. 16(3): p. 165-77.

10. Morganti-Kossmann, M.C., et al., Modulation of immune response by head injury. Injury, 2007. 38(12): p. 1392-400.

11. Davis, A.E., Mechanisms of traumatic brain injury: biomechanical, structural and cellular considerations. Crit Care Nurs Q, 2000. 23(3): p. 1-13.

12. Marklund, N., et al., Energy metabolic changes in the early post-injury period following traumatic brain injury in rats. Neurochem Res, 2006. 31(8): p. 1085-93.

13. Donat, C.K., et al., Microglial Activation in Traumatic Brain Injury. Front Aging Neurosci, 2017. 9: p. 208.

14. Russo, M.V. and D.B. McGavern, Inflammatory neuroprotection following traumatic brain injury. Science, 2016. 353(6301): p. 783-5.

15. Finnie, J.W., Neuroinflammation: beneficial and detrimental effects after traumatic brain injury. Inflammopharmacology, 2013. 21(4): p. 309-20.

16. Corps, K.N., T.L. Roth, and D.B. McGavern, Inflammation and neuroprotection in traumatic brain injury. JAMA Neurol, 2015. 72(3): p. 355-62.

17. Simon, D.W., et al., The far-reaching scope of neuroinflammation after traumatic brain injury. Nat Rev Neurol, 2017. 13(3): p. 171-191.

18. Aungst, S.L., et al., Repeated mild traumatic brain injury causes chronic neuroinflammation, changes in hippocampal synaptic plasticity, and associated cognitive deficits. J Cereb Blood Flow Metab, 2014. 34(7): p. 1223-32.

19. Morganti-Kossmann, M.C., et al., Inflammatory response in acute traumatic brain injury: a double-edged sword. Curr Opin Crit Care, 2002. 8(2): p. 101-5.

20. Jassam, Y.N., et al., Neuroimmunology of Traumatic Brain Injury: Time for a Paradigm Shift. Neuron, 2017. 95(6): p. 1246-1265.

21. Bellen, H.J., C. Tong, and $\mathrm{H}$. Tsuda, 100 years of Drosophila research and its impact on vertebrate neuroscience: a history lesson for the future. Nat Rev Neurosci, 2010. 11(7): p. 514-22.

22. Marsh, J.L. and L.M. Thompson, Drosophila in the study of neurodegenerative disease. Neuron, 2006. 52(1): p. 169-78.

23. Casci, I. and U.B. Pandey, A fruitful endeavor: modeling ALS in the fruit fly. Brain Res, 2015. 1607: p. 47-74.

24. Bouleau, S. and H. Tricoire, Drosophila models of Alzheimer's disease: advances, limits, and perspectives. J Alzheimers Dis, 2015. 45(4): p. 1015-38. 
769

770

771

772

773

774

775

776

777

778

779

780

781

782

783

784

785

786

787

788

789

790

791

792

793

794

795

796

797

798

799

800

801

802

803

804

805

806

807

808

809

810

811

812

813

814

815

816

817

818

25. Lewis, E.A. and G.A. Smith, Using Drosophila models of Huntington's disease as a translatable tool. J Neurosci Methods, 2016. 265: p. 89-98.

26. West, R.J., et al., Neurophysiology of Drosophila models of Parkinson's disease. Parkinsons Dis, 2015. 2015: p. 381281.

27. Allen, N.J. and B.A. Barres, Neuroscience: Glia - more than just brain glue. Nature, 2009. 457(7230): p. 675-7.

28. Chung, W.S., et al., Do glia drive synaptic and cognitive impairment in disease? Nat Neurosci, 2015. 18(11): p. 1539-1545.

29. Doherty, J., et al., Ensheathing glia function as phagocytes in the adult Drosophila brain. J Neurosci, 2009. 29(15): p. 4768-81.

30. Logan, M.A. and M.R. Freeman, The scoop on the fly brain: glial engulfment functions in Drosophila. Neuron Glia Biol, 2007. 3(1): p. 63-74.

31. Logan, M.A., et al., Negative regulation of glial engulfment activity by Draper terminates glial responses to axon injury. Nat Neurosci, 2012. 15(5): p. 722-30.

32. Ziegenfuss, J.S., et al., Draper-dependent glial phagocytic activity is mediated by Src and Syk family kinase signalling. Nature, 2008. 453(7197): p. 935-9.

33. Losada-Perez, M., Glia: from 'just glue' to essential players in complex nervous systems: a comparative view from flies to mammals. J Neurogenet, 2018. 32(2): p. 78-91.

34. Sonnenfeld, M.J. and J.R. Jacobs, Macrophages and glia participate in the removal of apoptotic neurons from the Drosophila embryonic nervous system. J Comp Neurol, 1995. 359(4): p. 644-52.

35. Purice, M.D., et al., A novel Drosophila injury model reveals severed axons are cleared through a Draper/MMP-1 signaling cascade. Elife, 2017. 6.

36. Lemaitre, B. and J. Hoffmann, The host defense of Drosophila melanogaster. Annu Rev Immunol, 2007. 25: p. 697-743.

37. Sudmeier, L.J., et al., Persistent Activation of the Innate Immune Response in Adult Drosophila Following Radiation Exposure During Larval Development. G3 (Bethesda), 2015. 5(11): p. 2299-306.

38. Petersen, A.J., S.A. Rimkus, and D.A. Wassarman, ATM kinase inhibition in glial cells activates the innate immune response and causes neurodegeneration in Drosophila. Proc Natl Acad Sci U S A, 2012. 109(11): p. E656-64.

39. Petersen, A.J., R.J. Katzenberger, and D.A. Wassarman, The innate immune response transcription factor relish is necessary for neurodegeneration in a Drosophila model of ataxia-telangiectasia. Genetics, 2013. 194(1): p. 133-42.

40. Katzenberger, R.J., et al., A Drosophila model of closed head traumatic brain injury. Proc Natl Acad Sci U S A, 2013. 110(44): p. E4152-9.

41. Barekat, A., et al., Using Drosophila as an integrated model to study mild repetitive traumatic brain injury. Sci Rep, 2016. 6: p. 25252.

42. Huang, Y., et al., Translational profiling of clock cells reveals circadianly synchronized protein synthesis. PLoS Biol, 2013. 11(11): p. e1001703.

43. Nichols, C.D., J. Becnel, and U.B. Pandey, Methods to assay Drosophila behavior. J Vis Exp, 2012(61).

44. Shaw, P.J., et al., Correlates of sleep and waking in Drosophila melanogaster. Science, 2000. 287(5459): p. 1834-7.

45. Huber, R., et al., Sleep homeostasis in Drosophila melanogaster. Sleep, 2004. 27(4): p. 628-39.

46. Linford, N.J., et al., Measurement of lifespan in Drosophila melanogaster. J Vis Exp, 2013(71).

47. Cardillo, G. LogRank: Comparing survival curves of two groups using the log rank test. http://www.mathworks.com/matlabcentral/fileexchange/22317 2008. 
819

820

821

822

823

824

825

826

827

828

829

830

831

832

833

834

835

836

837

838

839

840

841

842

843

844

845

846

847

848

849

850

851

852

853

854

855

856

857

858

859

860

861

862

863

864

865

866

867

868

869

48. Heiman, M., et al., Cell type-specific mRNA purification by translating ribosome affinity purification (TRAP). Nat Protoc, 2014. 9(6): p. 1282-91.

49. Nagoshi, E., et al., Dissecting differential gene expression within the circadian neuronal circuit of Drosophila. Nat Neurosci, 2010. 13(1): p. 60-8.

50. Bray, N.L., et al., Near-optimal probabilistic RNA-seq quantification. Nat Biotechnol, 2016. 34(5): p. 525-7.

51. Gramates, L.S., et al., FlyBase at 25: looking to the future. Nucleic Acids Res, 2017. 45(D1): p. D663-D671.

52. Soneson, C., M.I. Love, and M.D. Robinson, Differential analyses for RNA-seq: transcript-level estimates improve gene-level inferences. F1000Res, 2015. 4: p. 1521.

53. Love, M.I., W. Huber, and S. Anders, Moderated estimation of fold change and dispersion for RNA-seq data with DESeq2. Genome Biol, 2014. 15(12): p. 550.

54. Risso, D., et al., Normalization of RNA-seq data using factor analysis of control genes or samples. Nat Biotechnol, 2014. 32(9): p. 896-902.

55. Huang, D.W., et al., The DAVID Gene Functional Classification Tool: a novel biological module-centric algorithm to functionally analyze large gene lists. Genome Biol, 2007. 8(9): p. R183.

56. Huang, D.W., et al., DAVID Bioinformatics Resources: expanded annotation database and novel algorithms to better extract biology from large gene lists. Nucleic Acids Res, 2007. 35(Web Server issue): p. W169-75.

57. Bullard, J.H., et al., Evaluation of statistical methods for normalization and differential expression in mRNA-Seq experiments. BMC Bioinformatics, 2010. 11: p. 94.

58. Mouzon, B., et al., Repetitive mild traumatic brain injury in a mouse model produces learning and memory deficits accompanied by histological changes. J Neurotrauma, 2012. 29(18): p. 2761-73.

59. Krauss, J.K.J., J., Movement disorders after TBI. Brain Injury Medicine: Principles and Practice, ed. N.D.K. Zasler, D.I.; Zafonte, R.D. 2007, New York. 469-489.

60. Ustinova, K.I., et al., Physical therapy for correcting postural and coordination deficits in patients with mild-to-moderate traumatic brain injury. Physiother Theory Pract, 2015. 31(1): p. 1-7.

61. Guskiewicz, K.M., Balance assessment in the management of sport-related concussion. Clin Sports Med, 2011. 30(1): p. 89-102, ix.

62. Fujimoto, S.T., et al., Motor and cognitive function evaluation following experimental traumatic brain injury. Neurosci Biobehav Rev, 2004. 28(4): p. 365-78.

63. Hirsch, J. and L. Erlenmeyer-Kimling, Sign of taxis as a property of the genotype. Science, 1961. 134(3482): p. 835-6.

64. Feany, M.B. and W.W. Bender, A Drosophila model of Parkinson's disease. Nature, 2000. 404(6776): p. 394-8.

65. Barone, M.C. and D. Bohmann, Assessing neurodegenerative phenotypes in Drosophila dopaminergic neurons by climbing assays and whole brain immunostaining. J Vis Exp, 2013(74): p. e50339.

66. Ali, Y.O., et al., Assaying locomotor, learning, and memory deficits in Drosophila models of neurodegeneration. J Vis Exp, 2011(49).

67. McCall, K., J.S. Peterson, and T.L. Pritchett, Detection of cell death in Drosophila. Methods Mol Biol, 2009. 559: p. 343-56.

68. Sun, Y., et al., TRPA channels distinguish gravity sensing from hearing in Johnston's organ. Proc Natl Acad Sci U S A, 2009. 106(32): p. 13606-11.

69. Kamikouchi, A., et al., The neural basis of Drosophila gravity-sensing and hearing. Nature, 2009. 458(7235): p. 165-71.

70. Mettang, M., et al., IKK2/NF-kappaB signaling protects neurons after traumatic brain injury. FASEB J, 2018. 32(4): p. 1916-1932. 
870

871

872

873

874

875

876

877

878

879

880

881

882

883

884

885

886

887

888

889

890

891

892

893

894

895

896

897

898

899

900

901

902

903

904

905

906

907

908

909

910

911

912

913

914

915

916

917

918

919

920

71. Sandsmark, D.K., J.E. Elliott, and M.M. Lim, Sleep-Wake Disturbances After Traumatic Brain Injury: Synthesis of Human and Animal Studies. Sleep, 2017. 40(5).

72. Willie, J.T., et al., Controlled cortical impact traumatic brain injury acutely disrupts wakefulness and extracellular orexin dynamics as determined by intracerebral microdialysis in mice. J Neurotrauma, 2012. 29(10): p. 1908-21.

73. Lim, M.M., et al., Dietary therapy mitigates persistent wake deficits caused by mild traumatic brain injury. Sci Transl Med, 2013. 5(215): p. 215ra173.

74. Rowe, R.K., et al., Diffuse brain injury induces acute post-traumatic sleep. PLoS One, 2014. 9(1): p. e82507.

75. Morawska, M.M., et al., Sleep Modulation Alleviates Axonal Damage and Cognitive Decline after Rodent Traumatic Brain Injury. J Neurosci, 2016. 36(12): p. 3422-9.

76. Thomasy, H., Tumor Necrosis Factor alpha as a Potential Mediator of the Effects of Phosphodiesterase 4B Inhibition on Cognition after Traumatic Brain Injury. J Neurosci, 2016. 36(46): p. 11587-11589.

77. Hazra, A., et al., Delayed thalamic astrocytosis and disrupted sleep-wake patterns in a preclinical model of traumatic brain injury. J Neurosci Res, 2014. 92(11): p. 1434-45.

78. Petraglia, A.L., et al., The spectrum of neurobehavioral sequelae after repetitive mild traumatic brain injury: a novel mouse model of chronic traumatic encephalopathy. $\mathrm{J}$ Neurotrauma, 2014. 31(13): p. 1211-24.

79. Skopin, M.D., et al., Chronic decrease in wakefulness and disruption of sleep-wake behavior after experimental traumatic brain injury. J Neurotrauma, 2015. 32(5): p. 28996.

80. Thomas, J., et al., Identification of an intronic splicing regulatory element involved in auto-regulation of alternative splicing of SCL33 pre-mRNA. Plant J, 2012. 72(6): p. 93546.

81. Xiong, W.C., et al., repo encodes a glial-specific homeo domain protein required in the Drosophila nervous system. Genes Dev, 1994. 8(8): p. 981-94.

82. Huang, Y., F.S. Ng, and F.R. Jackson, Comparison of larval and adult Drosophila astrocytes reveals stage-specific gene expression profiles. G3 (Bethesda), 2015. 5(4): p. 551-8.

83. Auld, V.J., et al., Gliotactin, a novel transmembrane protein on peripheral glia, is required to form the blood-nerve barrier in Drosophila. Cell, 1995. 81(5): p. 757-67.

84. Bainton, R.J., et al., moody encodes two GPCRs that regulate cocaine behaviors and blood-brain barrier permeability in Drosophila. Cell, 2005. 123(1): p. 145-56.

85. Benjamini, Y.H., Y, Controlling the false discovery rate: a practical and powerful approach to multiple testing. Journal of the Royal Statistical Society, Series B, 1995. 57(1): p. 289-300.

86. Ekengren, S. and D. Hultmark, A family of Turandot-related genes in the humoral stress response of Drosophila. Biochem Biophys Res Commun, 2001. 284(4): p. 998-1003.

87. Imler, J.L. and P. Bulet, Antimicrobial peptides in Drosophila: structures, activities and gene regulation. Chem Immunol Allergy, 2005. 86: p. 1-21.

88. Ng, F.S., et al., TRAP-seq Profiling and RNAi-Based Genetic Screens Identify Conserved Glial Genes Required for Adult Drosophila Behavior. Front Mol Neurosci, 2016. 9: p. 146.

89. Buchon, N., N. Silverman, and S. Cherry, Immunity in Drosophila melanogaster--from microbial recognition to whole-organism physiology. Nat Rev Immunol, 2014. 14(12): p. 796-810.

90. De Gregorio, E., et al., Genome-wide analysis of the Drosophila immune response by using oligonucleotide microarrays. Proc Natl Acad Sci U S A, 2001. 98(22): p. 12590-5.

91. Cerenius, L. and K. Soderhall, Coagulation in invertebrates. J Innate Immun, 2011. 3(1): p. 3-8. 
921

922

923

924

925

926

927

928

929

930

931

932

933

934

935

936

937

938

939

940

941

942

943

944

945

946

947

948

949

950

951

952

953

954

955

956

957

958

959

960

961

962

963

964

965

966

967

968

969

970

971

92. Kambris, Z., et al., Drosophila immunity: a large-scale in vivo RNAi screen identifies five serine proteases required for Toll activation. Curr Biol, 2006. 16(8): p. 808-13.

93. Lemaitre, B., et al., The dorsoventral regulatory gene cassette spatzle/Toll/cactus controls the potent antifungal response in Drosophila adults. Cell, 1996. 86(6): p. 97383.

94. Verma, R.P. and C. Hansch, Matrix metalloproteinases (MMPs): chemical-biological functions and (Q)SARs. Bioorg Med Chem, 2007. 15(6): p. 2223-68.

95. Welch, W.J., Mammalian stress response: cell physiology, structure/function of stress proteins, and implications for medicine and disease. Physiol Rev, 1992. 72(4): p. 106381.

96. Chen, J., et al., Participation of the $p 38$ pathway in Drosophila host defense against pathogenic bacteria and fungi. Proc Natl Acad Sci U S A, 2010. 107(48): p. 20774-9.

97. Kume, K., et al., Dopamine is a regulator of arousal in the fruit fly. J Neurosci, 2005. 25(32): p. 7377-84.

98. Wu, M.N., et al., A genetic screen for sleep and circadian mutants reveals mechanisms underlying regulation of sleep in Drosophila. Sleep, 2008. 31(4): p. 465-72.

99. Ueno, T. and K. Kume, Functional characterization of dopamine transporter in vivo using Drosophila melanogaster behavioral assays. Front Behav Neurosci, 2014. 8: p. 303.

100. Pearson, J.C., et al., Multiple transcription factor codes activate epidermal woundresponse genes in Drosophila. Proc Natl Acad Sci U S A, 2009. 106(7): p. 2224-9.

101. Gaudet, P.L., M.; Thomas, P., Gene Ontology annotation inferences using phylogenetic trees. GO Reference Genome Project, 2010. http://www.geneontology.org/cgibin/references.cgi\#GO_REF0000033.

102. Oeckinghaus, A., M.S. Hayden, and S. Ghosh, Crosstalk in NF-kappaB signaling pathways. Nat Immunol, 2011. 12(8): p. 695-708.

103. Mattson, M.P., NF-kappaB in the survival and plasticity of neurons. Neurochem Res, 2005. 30(6-7): p. 883-93.

104. Helmy, A., et al., The cytokine response to human traumatic brain injury: temporal profiles and evidence for cerebral parenchymal production. J Cereb Blood Flow Metab, 2011. 31(2): p. 658-70.

105. Bales, K.R., et al., The NF-kappaB/Rel family of proteins mediates Abeta-induced neurotoxicity and glial activation. Brain Res Mol Brain Res, 1998. 57(1): p. 63-72.

106. Brambilla, R., et al., Inhibition of astroglial nuclear factor kappaB reduces inflammation and improves functional recovery after spinal cord injury. J Exp Med, 2005. 202(1): p. 145-56.

107. Yang, K., X.S. Mu, and R.L. Hayes, Increased cortical nuclear factor-kappa B (NF-kappa B) DNA binding activity after traumatic brain injury in rats. Neurosci Lett, 1995. 197(2): $\mathrm{p}$. 101-4.

108. Nonaka, M., et al., Prolonged activation of NF-kappaB following traumatic brain injury in rats. J Neurotrauma, 1999. 16(11): p. 1023-34.

109. Hu, Y.C., et al., Biphasic activation of nuclear factor kappa $B$ and expression of $p 65$ and c-Rel after traumatic brain injury in rats. Inflamm Res, 2014. 63(2): p. 109-15.

110. Khush, R.S., F. Leulier, and B. Lemaitre, Drosophila immunity: two paths to NF-kappaB. Trends Immunol, 2001. 22(5): p. 260-4.

111. Kuo, T.H., et al., Sleep triggered by an immune response in Drosophila is regulated by the circadian clock and requires the NFkappaB Relish. BMC Neurosci, 2010. 11: p. 17.

112. Cao, Y., et al., Dnr1 mutations cause neurodegeneration in Drosophila by activating the innate immune response in the brain. Proc Natl Acad Sci U S A, 2013. 110(19): $p$. E1752-60.

113. Kounatidis, I., et al., NF-kappaB Immunity in the Brain Determines Fly Lifespan in Healthy Aging and Age-Related Neurodegeneration. Cell Rep, 2017. 19(4): p. 836-848. 
972

973

974

975

976

977

978

979

980

981

982

983

984

985

986

987

988

989

990

991

992

993

994

995

996

997

998

999

1000

1001

1002

1003

1004

1005

1006

1007

1008

1009

1010
114. Hayden, M.S. and S. Ghosh, NF-kappaB, the first quarter-century: remarkable progress and outstanding questions. Genes Dev, 2012. 26(3): p. 203-34.

115. Hetru, C. and J.A. Hoffmann, NF-kappaB in the immune response of Drosophila. Cold Spring Harb Perspect Biol, 2009. 1(6): p. a000232.

116. Sun, M. and L.L. Chen, A Novel Method to Model Chronic Traumatic Encephalopathy in Drosophila. J Vis Exp, 2017(125).

117. Bartholomew, N.R., et al., Impaired climbing and flight behaviour in Drosophila melanogaster following carbon dioxide anaesthesia. Sci Rep, 2015. 5: p. 15298.

118. Statler, K.D., et al., Isoflurane improves long-term neurologic outcome versus fentanyl after traumatic brain injury in rats. J Neurotrauma, 2000. 17(12): p. 1179-89.

119. Statler, K.D., et al., Comparison of seven anesthetic agents on outcome after experimental traumatic brain injury in adult, male rats. J Neurotrauma, 2006. 23(1): p. 97-108.

120. Statler, K.D., et al., Isoflurane exerts neuroprotective actions at or near the time of severe traumatic brain injury. Brain Res, 2006. 1076(1): p. 216-24.

121. Fischer, J.A., et al., Anesthetics Influence Mortality in a Drosophila Model of Blunt Trauma With Traumatic Brain Injury. Anesth Analg, 2018. 126(6): p. 1979-1986.

122. Graber, D.J., B.A. Costine, and W.F. Hickey, Early inflammatory mediator gene expression in two models of traumatic brain injury: ex vivo cortical slice in mice and in vivo cortical impact in piglets. J Neuroinflammation, 2015. 12: p. 76.

123. Morganti, J.M., L.K. Riparip, and S. Rosi, Call Off the Dog(ma): M1/M2 Polarization Is Concurrent following Traumatic Brain Injury. PLoS One, 2016. 11(1): p. e0148001.

124. Lagraoui, M., et al., Controlled cortical impact and craniotomy induce strikingly similar profiles of inflammatory gene expression, but with distinct kinetics. Front Neurol, 2012. 3: p. 155.

125. Meng, Q., et al., Traumatic Brain Injury Induces Genome-Wide Transcriptomic, Methylomic, and Network Perturbations in Brain and Blood Predicting Neurological Disorders. EBioMedicine, 2017. 16: p. 184-194.

126. Almeida-Suhett, C.P., et al., Temporal course of changes in gene expression suggests a cytokine-related mechanism for long-term hippocampal alteration after controlled cortical impact. J Neurotrauma, 2014. 31(7): p. 683-90.

127. Williams, J.A., et al., Interaction between sleep and the immune response in Drosophila: a role for the NFkappaB relish. Sleep, 2007. 30(4): p. 389-400.

128. Kuo, T.H., A. Handa, and J.A. Williams, Quantitative measurement of the immune response and sleep in Drosophila. J Vis Exp, 2012(70): p. e4355.

129. Nappi, A.J. and E. Vass, Melanogenesis and the generation of cytotoxic molecules during insect cellular immune reactions. Pigment Cell Res, 1993. 6(3): p. 117-26. 